\title{
Effect of anisotropic scattering for rotational collisions on electron transport parameters in $\mathrm{CO}$
}

Citation for published version (APA):

Vialetto, L., Ben Moussa, A., van Dijk, J., Longo, S., Diomede, P., Guerra, V., \& Alves, L. L. (2021). Effect of anisotropic scattering for rotational collisions on electron transport parameters in CO. Plasma Sources Science \& Technology, 30(7), [075001]. https://doi.org/10.1088/1361-6595/ac0a4d

Document status and date:

Published: 01/07/2021

DOI:

10.1088/1361-6595/ac0a4d

Document Version:

Publisher's PDF, also known as Version of record

Document license:

Taverne

Please check the document version of this publication:

- A submitted manuscript is the version of the article upon submission and before peer-review. There can be important differences between the submitted version and the official published version of record.

People interested in the research are advised to contact the author for the final version of the publication, or visit the DOI to the publisher's website.

- The final author version and the galley proof are versions of the publication after peer review.

- The final published version features the final layout of the paper including the volume, issue and page numbers.

Link to publication

\footnotetext{
General rights rights.

- You may freely distribute the URL identifying the publication in the public portal. please follow below link for the End User Agreement:

www.umlib.nl/taverne-license

Take down policy

If you believe that this document breaches copyright please contact us at:

repository@maastrichtuniversity.nl

providing details and we will investigate your claim.
}

Copyright and moral rights for the publications made accessible in the public portal are retained by the authors and/or other copyright owners and it is a condition of accessing publications that users recognise and abide by the legal requirements associated with these

- Users may download and print one copy of any publication from the public portal for the purpose of private study or research.

- You may not further distribute the material or use it for any profit-making activity or commercial gain

If the publication is distributed under the terms of Article $25 \mathrm{fa}$ of the Dutch Copyright Act, indicated by the "Taverne" license above, 


\title{
Effect of anisotropic scattering for rotational collisions on electron transport parameters in $\mathrm{CO}$
}

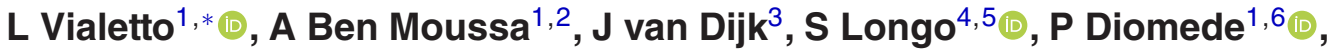 \\ V Guerra ${ }^{7}$ (i) and L L Alves ${ }^{7}$ (i) \\ ${ }^{1}$ Center for Computational Energy Research, DIFFER-Dutch Institute for Fundamental Energy Research, \\ De Zaale 20, 5612 AJ Eindhoven, The Netherlands \\ 2 Mines ParisTech, Boulevard Saint-Michel 60, 75272 Paris, France \\ 3 Department of Applied Physics, Eindhoven University of Technology, PO Box 513, 5600 MB \\ Eindhoven, The Netherlands \\ ${ }^{4}$ Dipartimento di Chimica, Università degli Studi di Bari, via Orabona 4, 70126 Bari, Italy \\ ${ }^{5}$ Istituto per la Scienza e Tecnologia dei Plasmi, CNR, 70126 Bari, Italy \\ ${ }^{6}$ Faculty of Science and Engineering, Maastricht University, Paul Henri Spaaklaan 1, 6229 GS \\ Maastricht, The Netherlands \\ ${ }^{7}$ Instituto de Plasmas e Fusão Nuclear, Instituto Superior Técnico, Universidade de Lisboa, Av. Rovisco \\ Pais 1, 1049-001 Lisboa, Portugal \\ E-mail: L.Vialetto@ differ.nl
}

Received 26 February 2021, revised 28 May 2021

Accepted for publication 10 June 2021

Published 1 July 2021

\begin{abstract}
The role of anisotropic scattering in rotational collisions of electrons with $\mathrm{CO}$ molecules is investigated numerically with Monte Carlo (MC) simulations and with calculations using the Lisbon KInetics two-term Boltzmann solver (LoKI-B). The study adopts integral cross sections taken from the IST-Lisbon database of LXCat or extracted from Biagi's code Magboltz v11.10. Different angular scattering models for rotational collisions are implemented and compared in MC simulations, and a novel anisotropic scattering model is derived from the dipole-Born differential cross sections, to describe the strongly forward-peaked nature of rotational collisions. This model is also implemented in LoKI-B, to describe the anisotropic inelastic/superelastic scattering in dipole rotational collisions, using coherent expressions for the corresponding integral and momentum transfer cross sections. The comparison between MC and LoKI-B results shows that the calculation of swarm parameters is more influenced by the choice of the angular scattering model than the adoption of the two-term approximation, yielding deviations up to $50 \%$ in the reduced mobility for different angular distributions. The consequences in the swarm derivation of cross sections are also discussed. Finally, it is shown that inclusion of electric-quadrupole interactions, usually neglected in electron swarm studies, can improve the agreement between numerical results and measurements.
\end{abstract}

Keywords: carbon monoxide, anisotropic scattering, angular distribution, cross section

S. Supplementary material for this article is available online

(Some figures may appear in colour only in the online journal)

* Author to whom any correspondence should be addressed. 


\section{Introduction}

Carbon monoxide ( $\mathrm{CO}$ ) plays an important role in our atmosphere and in the interstellar medium, a role that is also fundamental in a wide variety of plasma applications, such as plasma deposition [1] and high power lasers [2]. Detailed cross sections for interactions of electrons with $\mathrm{CO}$ are needed in areas such as reforming of $\mathrm{CO}_{2}[3,4]$, studies of $\mathrm{CO}$ adsorption on surfaces [5] and re-entry physics [6]. For instance, the CO Cameron bands ( $a^{3} \Pi \rightarrow X^{1} \Sigma$ ) were observed in the emission spectra of the Martian atmosphere [7] and in atmospheric convection in Jupiter [8]. Electron scattering by $\mathrm{CO}$ is also relevant in astrophysics, since this molecule is the most abundant in molecular clouds, after hydrogen. In particular, rotational excitation of $\mathrm{CO}$ by photoelectrons and subsequent emission of radiation are important processes in the energy balance of astrophysical plasmas [9]. The electron energy range for those phenomena goes down to tens of meV [1].

Several reviews of binary collisions data and swarm parameters of electrons in $\mathrm{CO}$ can be found in literature. As an example, a comprehensive review of experimental methods and electron scattering data published until 1983 is given by Trajmar and co-authors [10]. An updated version has been published by Brunger and Buckman [11] accounting for measurements until 2002 and later by Raju [1]. Those cross sections cannot be used directly in plasma modelling, since they do not constitute a complete set, as the ones typically derived from swarm analysis [12]. Examples of swarm-derived cross sections for electrons in $\mathrm{CO}$ are the ones by Hake and Phelps [13] and by Land [14]. A complete review of electron impact cross sections in $\mathrm{CO}$ has also been performed by Itikawa [15]. Phelps and Itikawa cross sections sets are available in table format at LXCat databases [16, 17].

Recently, a complete set of cross sections for electron scattering in CO has been proposed by the Lisbon group [18] and it is available at the IST-Lisbon database of LXCat [19]. This set has been optimized by comparing results of transport parameters calculated using the Lisbon KInetics two-term Boltzmann solver (LoKI-B) [20] with experimental measurements at different gas temperatures $\left(T_{\text {gas }}\right)$ and reduced electric fields $(E / N)$. One important contribution of the work by the Lisbon group [18] is the analysis of cross sections at low energies, where the electron-dipole interaction dominates. In [18], the importance of rotational excitation and de-excitation processes for calculations of electron swarm parameters is highlighted. Moreover, these authors propose an elastic momentum transfer (MT) cross section for collisions of electrons with the ground electronic state of $\mathrm{CO}$, from which one can estimate a total MT.

The approach recommended by the Lisbon group is different than the one adopted by Hake and Phelps [13], where a total MT cross section is used directly in the calculations. More specifically, the total MT determined in the analysis by Hake and Phelps [13] is given by the sum of a dipole term, calculated as a rotational MT with the Born approximation [21], and a nitrogen-like elastic MT cross section. In the same work, the effect of 16 different rotational levels of CO is also analysed. In addition, in [13], a novel approach was investigated, where the effect of several rotational levels was approximated by a single rotational level. Nevertheless, cross sections for rotational collisions are not reported either in the Phelps [16] or in the Itikawa [17] database of LXCat.

Building upon the results by the Lisbon group [18], the present work extends the analysis of that cross sections set, focussing on the description of rotational collisions. Specifically, this work is motivated by the discrepancies found in electron transport parameters obtained from two-term solutions of the electron Boltzmann equation using the IST-Lisbon dataset and experimental measurements. Indeed, deviations between measurements and calculations are up to $20 \%$ at $77 \mathrm{~K}$, exceeding the experimental error, typically below 3\%. This problem is also relevant for plasma modelling, since uncertainties around $10 \%$ are typically taken as acceptable upper limit for transport coefficients [12]. Moreover, the accuracy of the two-term approximation for optimization of electron impact cross sections in $\mathrm{CO}$ has not been addressed in previous studies by Hake and Phelps [13] and Ogloblina and co-authors [18].

In this work, Monte Carlo (MC) calculations of electron transport parameters in $\mathrm{CO}$ are compared to numerical results obtained with the two-term solver LoKI-B [20] and with experimental measurements, to test the accuracy of the two-term approximation. A fully native MC simulations code developed recently by some of the present authors is used [22, 23]. Moreover, two different datasets of cross sections are taken into account: the IST-Lisbon one available at LXCat [19] and the one from Biagi's code Magboltz v11.10 [24]. To the best of our knowledge, Biagi's cross sections form the only complete set available for use in an MC simulations code and this work presents a first independent analysis of this dataset for calculations of electron transport parameters. Particular emphasis is given to (i) the angular model adopted to describe anisotropic scattering in rotational collisions and (ii) the inclusion of this effect in both MC and two-term codes.

In the pioneering work by Reid [25], the assumption of isotropic scattering was tested in a number of different model gases, with two-term solutions and MC simulations. Similarly, the inclusion of anisotropic scattering in electron-molecule collisions in nitrogen was considered by Phelps and Pitchford [26], using a generalization of the traditional two-term expansion of the electron velocity distribution function (EVDF) in Legendre polynomials. In [26], it has been found that the changes in chemical rate coefficients and transport parameters due to the inclusion of anisotropic scattering are smaller than $1 \%$ in the range $10 \leqslant E / N \leqslant 50 \mathrm{Td}$ and up to $10 \%$ at higher $E / N$ values, between 500 and $1500 \mathrm{Td}$. Recently, a comparison of different anisotropic scattering models in MC simulations was made by Janssen and co-authors [27]. They recommend forward inelastic scattering for an argon-like model gas and, in general, whenever differential cross sections (DCSs) are not available. However, their model does not consider the role of low-energy threshold excitations that are typical of molecular gases, like $\mathrm{CO}$.

Casey and co-authors analysed the effect of anisotropic scattering for rotational collisions of electrons in molecular nitrogen, using a two-term and a multi-term solver [28]. They found that the inclusion of the anisotropic contributions 
changes the drift velocity up to $11 \%$ and $10 \%$ at $77 \mathrm{~K}$ and $293 \mathrm{~K}$, respectively, compared with the isotropic case. This effect decreases with increasing $E / N$, for both temperatures. However, in $\mathrm{N}_{2}$ only electric quadrupole processes are present, whereas electron scattering with $\mathrm{CO}$ molecules is dominated by dipole interactions at low energies. In fact, the description of different types of rotational interactions requires different angular scattering models that are consistent with the net momentum exchange. This aspect is not addressed in the work of Casey and co-authors [28], where simple empirical formulas are used for anisotropic rotational scattering, similar to the ones proposed by Kushner [29] and by Reid [25].

Here, we conduct a detailed study on low-energy threshold anisotropic collisions in $\mathrm{CO}$ and their impact on electron swarm parameters, assessing different angular scattering models for dipole rotational collisions. We propose a novel model to describe the strongly forward-peaked nature of electrondipole interactions, based on the Born approximation, for use in MC simulations codes. This anisotropic scattering treatment is included also in the two-term code LoKI-B [20]. The inclusion of this treatment is consistent with previous treatments by Phelps and Pitchford [26] and Pitchford et al [30], but it was neglected in [18].

The paper is organised as follows. In section 2, the key equations describing the scattering in dipole rotational collisions are introduced. In section 3, the electron impact cross sections from Biagi's Magboltz code v11.10 [24] are described and compared with the IST-Lisbon dataset [18]. Different angular scattering models for electron-dipole interactions are investigated in section 4 . Section 5 presents a comparison between measurements and calculations of electron swarm parameters in $\mathrm{CO}$, obtained with MC simulations and LoKI$\mathrm{B}$ solutions. Attention is given to the effect of different angular distribution models on MC calculations of transport coefficients. In section 5.2, the addition of quadrupole rotational collisions to Biagi's dataset is discussed. In section 6, the implementation of anisotropic inelastic scattering in the twoterm Boltzmann solver LoKI-B is discussed, highlighting the differences with the MC description. Section 7 summarizes the main results and concludes the paper.

\section{Electron scattering in dipole rotational collisions}

\subsection{Differential, integral and momentum transfer cross sections for dipole rotational collisions of electrons in $\mathrm{CO}$}

The kinematics of a scattering event is described by its DCS $\frac{\mathrm{d} \sigma(\epsilon, \theta)}{\mathrm{d} \Omega}$, where $\epsilon$ is the incident electron energy, $\theta$ is the polar scattering angle and $\mathrm{d} \Omega=2 \pi \sin \theta \mathrm{d} \theta$ is the differential solid angle, under the assumption of azimuthal symmetry. The DCS provides information about the angular distribution of electrons after the scattering. It is well known that, in the lowenergy region, rotational transitions can be described by the Born approximation combined with the point-dipole interaction [15]. In the particular case of polar molecules, like CO,

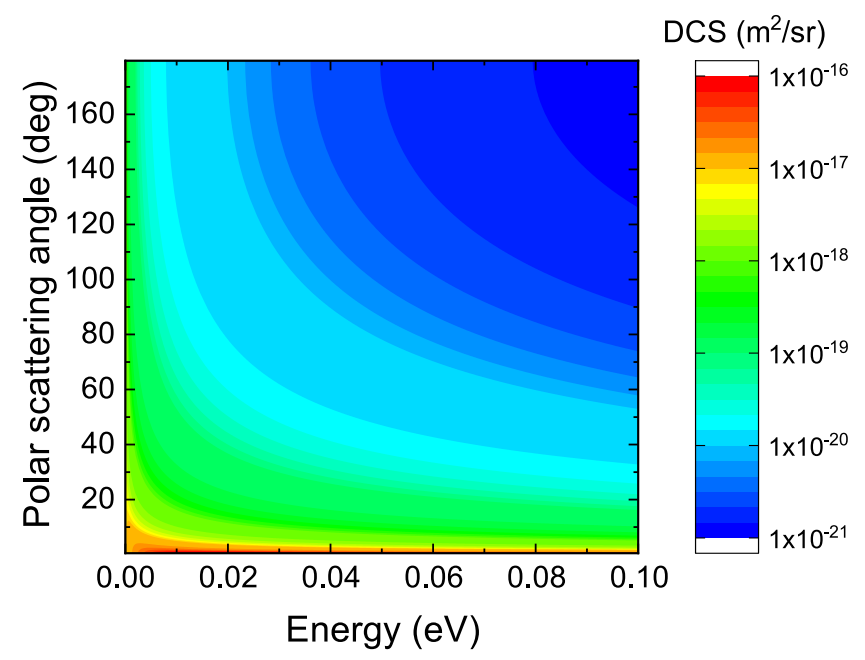

Figure 1. DCS from dipole-Born approximation, as a function of the incident electron energy and the polar scattering angle, for the $J=0 \rightarrow 1$ rotational excitation by electron impact in CO.

the DCS for rotational transitions is calculated as:

$$
\frac{\mathrm{d} \sigma(\epsilon, \theta)}{\mathrm{d} \Omega}=\frac{4}{3} \frac{\left(a_{0} \mu\right)^{2} R_{\infty}}{K^{2}} \sqrt{\frac{\epsilon^{\prime}}{\epsilon}} \frac{J_{>}}{2 J+1},
$$

where $a_{0}$ is the Bohr radius, $\mu=4.32 \times 10^{-2}$ is the permanent dipole moment of $\mathrm{CO}$ in atomic units, $R_{\infty}$ is the Rydberg constant in $\mathrm{eV}, \epsilon$ and $\epsilon^{\prime}$ are the electron energies before and after the collision, respectively, for transitions between states with rotational quantum numbers $J$ and $J^{\prime}$ with $J^{\prime}-J= \pm 1$, $J_{>}=\max \left(J, J^{\prime}\right)$ is the reduced matrix element of the transition $J \rightarrow J^{\prime}$, and $K^{2}=\left|\mathbf{k}^{\prime}-\mathbf{k}\right|^{2}=\epsilon^{\prime}+\epsilon-2 \sqrt{\epsilon \epsilon^{\prime}} \cos \theta$ is the squared magnitude of the change in relative momentum, due to the collision. Equation (1) is valid for linear molecules, like CO. More generally, other expressions are derived by Crawford for symmetric-top molecules [31]. The DCS given by equation (1) is plotted in figure 1, as a function of the incident electron energy and the polar scattering angle, for the transition from $J=0$ to $J^{\prime}=1$. In particular, due to the long-range dipole interaction, a highly anisotropic angular distribution is obtained, where small-angle scattering is dominant. Moreover, even if scattering at extreme angles (i.e. $\theta>90^{\circ}$ ) is not forbidden, this event is highly unlikely, due to the sharp decrease of the DCS at increasing angles. From this first consideration, it can be noted that dipole rotational collisions in the Born approximation do not lead to an isotropic angular scattering distribution function.

Integration of the DCS over the solid angle yields the integral cross section (ICS):

$$
\sigma^{\mathrm{ICS}}(\epsilon)=2 \pi \int_{0}^{\pi} \frac{\mathrm{d} \sigma(\epsilon, \theta)}{\mathrm{d} \Omega} \sin \theta \mathrm{d} \theta .
$$

In [32], ICSs for dipole rotational excitations $\sigma_{J, J+1}^{\mathrm{ICS}}(\epsilon)$ are obtained from equation (2), by direct integration of the DCS from equation (1) over the solid angle. These cross sections 
can be written as:

$$
\sigma_{J, J+1}^{\mathrm{ICS}}(\epsilon)=\frac{\left(a_{0} \mu\right)^{2} R_{\infty}}{V_{J, J+1}} \frac{J+1}{2 J+1} f\left(\epsilon / V_{J, J+1}\right),
$$

where $V_{J, J+1}$ is the energy threshold of the transition $J \rightarrow J+$ 1 in $\mathrm{eV}$ and

$$
f(x)=\frac{16 \pi}{3 x} \log (\sqrt{x}+\sqrt{x-1})
$$

is the reduced excitation cross section, with $x=\epsilon / V_{J, J+1}$.

In the pioneering works by Altshuler [21], Crawford [31], and Shimamura and Takayanagi [33], it has been shown that the electron-dipole interaction in the Born approximation can be described by a MT cross section that is generally different from the corresponding integral. In their work, the MT cross section is calculated as

$$
\sigma^{\mathrm{MT}}(\epsilon)=2 \pi \int_{0}^{\pi} \frac{\mathrm{d} \sigma(\epsilon, \theta)}{\mathrm{d} \Omega}(1-\cos \theta) \sin \theta \mathrm{d} \theta .
$$

From equations (1) and (5), the electron impact MT cross sections for transitions between the rotational levels $J$ and $J+1$ can be written as:

$$
\sigma_{J, J+1}^{\mathrm{MT}}(\epsilon)=\frac{\left(a_{0} \mu\right)^{2} R_{\infty}}{V_{J, J+1}} \frac{J+1}{2 J+1} g\left(\epsilon / V_{J, J+1}\right),
$$

where the function $g\left(\epsilon / V_{J, J+1}\right)$ is given by

$$
g(x)=\frac{8 \pi}{3 x}\left[1-\frac{(\sqrt{x}-\sqrt{x-1})^{2}}{\sqrt{x} \sqrt{x-1}} \log (\sqrt{x}+\sqrt{x-1})\right] .
$$

Strictly, equation (5) is valid for describing the MT of electrons in elastic collisions. More generally, an expression for inelastic MT cross sections was proposed by Makabe and White [34] and it can be written as

$$
\begin{aligned}
\sigma_{J, J+1}^{\mathrm{MT}}(\epsilon)= & 2 \pi \int_{0}^{\pi} \frac{\mathrm{d} \sigma(\epsilon, \theta)}{\mathrm{d} \Omega}\left[1-\left(1-\frac{V_{J, J+1}}{\epsilon}\right)^{1 / 2} \cos \theta\right] \\
& \times \sin \theta \mathrm{d} \theta
\end{aligned}
$$

where the term $\left[1-\left(1-\frac{V_{J, J+1}}{\epsilon}\right)^{1 / 2} \cos \theta\right]$ is the relative rate of MT for electrons in a two-body electron-molecule collision. Note that equation (8) is generally applicable to any kind of inelastic scattering, and not only to the case of dipole-rotational collisions. By substitution of equation (1) into equation (8), it can be verified that the electron impact MT cross section for the transition $J \rightarrow J+1$ has the same form of equation (6) where the function $g(x)$ of equation (7) is replaced by the following expression

$$
\begin{aligned}
g_{\text {corr }}(x)= & \frac{8 \pi}{3 x}\left\{\eta(x)-\frac{[\eta(x)(2 x-1)-2 \sqrt{x} \sqrt{x-1}]}{\sqrt{x} \sqrt{x-1}}\right. \\
& \times \log (\sqrt{x}+\sqrt{x-1})\},
\end{aligned}
$$

with $\eta(x)=\left(1-x^{-1}\right)^{1 / 2}$. In other words, equation (7) is a particular case of equation (9) that can be retrieved by setting

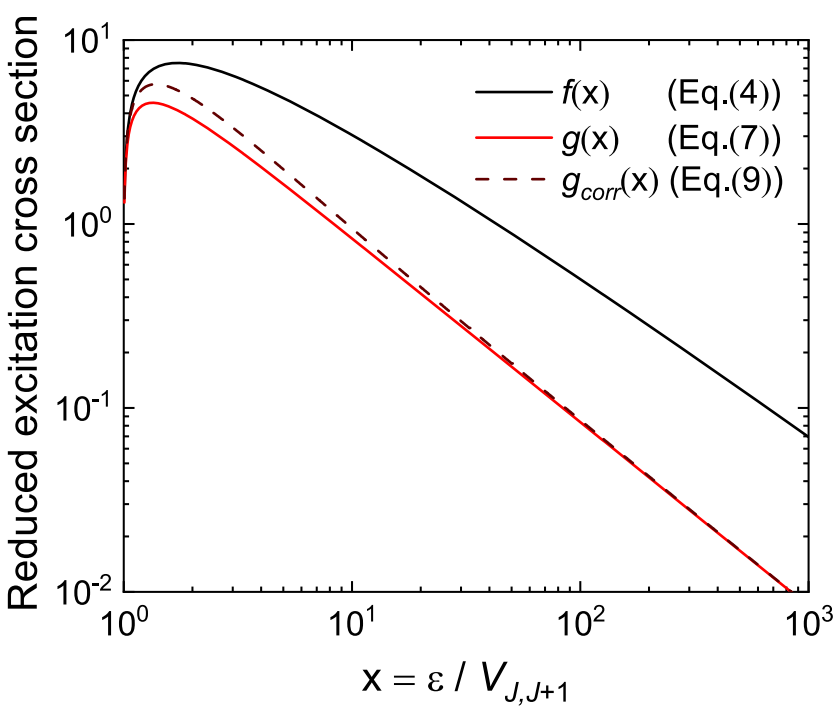

Figure 2. Reduced cross sections of dipole rotational excitations: integral contribution (solid black line), from equation (4), and momentum transfer contributions, from equation (7) (solid red line) and equation (9) (dashed line).

$\eta(x)=1$ or, equivalently, in the limit for $V_{J, J+1} / \epsilon \rightarrow 0$. Integral and MT reduced cross sections from equations (4), (7) and (9) are plotted in figure 2.

Small percent deviations between $g\left(\epsilon / V_{J, J+1}\right)$ and $g_{\text {corr }}\left(\epsilon / V_{J, J+1}\right)$ are found for $x \geqslant 20$, within $8 \%$. As expected, larger discrepancies appears in the limit $x \rightarrow 1$, when the incident electron energy is comparable with the energy threshold of rotational collisions. Nevertheless, the percent deviations for $x<20$ are still relatively small, up to $22 \%$ at $x=1.9$. Hence, the use of equations (5) and (7), as derived by Altshuler [21] for a linear molecule, is justified for dipole rotational collisions of electrons in $\mathrm{CO}$ when (i) the rotational energy difference between consecutive levels is sufficiently small, (ii) the point-dipole interaction is adopted, and (iii) the Born approximation is applied. The validity of such approximation for dipole rotational collisions is discussed in subsection 2.2. The use of equation (5), instead of equation (8), for dipole rotational collisions in a two-term Boltzmann solver is also justified in section 6. For other inelastic processes involving higher energy exchanges, the use of an appropriate expression of inelastic MT cross section, as defined in equation (8), is recommended.

Moreover, since $g\left(\epsilon / V_{J, J+1}\right)<f\left(\epsilon / V_{J, J+1}\right)$, each rotational MT cross section is smaller than the corresponding ICS. Taking into account the differences between $f\left(\epsilon / V_{J, J+1}\right)$ and $g\left(\epsilon / V_{J, J+1}\right)$ is important not only for an accurate description of the energy and MT rates in rotational collisions, but also to define a total (or effective) MT cross section, corresponding to the sum of the MT cross sections for all elastic/inelastic processes. In fact, equations (6) and (7) were used also by Hake and Phelps [13] for the description of the low energy part of their total MT cross section. In particular, the difference between $f\left(\epsilon / V_{J, J+1}\right)$ and $g\left(\epsilon / V_{J, J+1}\right)$, as shown in figure 2, can explain the apparent inconsistency found by Hake and Phelps [13], where the effective MT cross section is smaller than the 
total ICS for rotational collisions [18]. This inconsistency is solved by considering that the total MT cross section in the Phelps database of LXCat [16] is given, as a first approximation, by the sum of an elastic MT cross section with the rotational MT cross sections $\sigma_{J, J+1}^{\mathrm{MT}}(\epsilon)$, weighted by the fractional populations of the respective rotational levels. The justification for using $\sigma_{J, J+1}^{\mathrm{MT}}(\epsilon)$, instead of $\sigma_{J, J+1}^{\mathrm{ICS}}(\epsilon)$, to calculate the total MT cross section is further explained in section 6, where it is shown that this is a good approximation for the description of dipole rotational collisions also in a two-term Boltzmann solver.

Note that, with the detailed-balance assumption, the formula of Klein-Rosseland [35] can be used for calculations of de-excitation cross sections from the corresponding excitation ones as

$$
\sigma_{J+1, J}(\epsilon)=\frac{2 J+1}{2 J+3}\left(\frac{\epsilon+V_{J, J+1}}{\epsilon}\right) \sigma_{J, J+1}\left(\epsilon+V_{J, J+1}\right) .
$$

Hence, by substitution of equations (3) and (6) into equation (10), the ICS and MT cross section for de-excitation from the rotational level $J+1$ to $J$ can be written as

$$
\begin{aligned}
& \sigma_{J+1, J}^{\mathrm{ICS}}(\epsilon)=\frac{\left(a_{0} \mu\right)^{2} R_{\infty}}{V_{J, J+1}} \frac{J+1}{2 J+3} f_{\text {sup }}\left(\epsilon / V_{J, J+1}\right), \\
& \sigma_{J+1, J}^{\mathrm{MT}}(\epsilon)=\frac{\left(a_{0} \mu\right)^{2} R_{\infty}}{V_{J, J+1}} \frac{J+1}{2 J+3} g_{\text {sup }}\left(\epsilon / V_{J, J+1}\right),
\end{aligned}
$$

respectively. Functions $f_{\text {sup }}\left(\epsilon / V_{J, J+1}\right)$ and $g_{\text {sup }}\left(\epsilon / V_{J, J+1}\right)$ in equations (11) and (12) are

$$
\begin{gathered}
f_{\text {sup }}(x)=\frac{16 \pi}{3 x} \log (\sqrt{x+1}+\sqrt{x}) \\
g_{\text {sup }}(x)=\frac{8 \pi}{3 x}\left[1-\frac{(\sqrt{x+1}-\sqrt{x})^{2}}{\sqrt{x+1} \sqrt{x}} \log (\sqrt{x+1}+\sqrt{x})\right] .
\end{gathered}
$$

The same considerations on the differences between ICSs and MT cross sections for inelastic rotational collisions apply to superelastic collisions.

For the purpose of this study, the definitions of DCS (equation (1)), ICS (equations (3) and (4)) MT cross section (equations (6) and (7)) are important when comparing results from MC simulations and two-term calculations. On the one hand, as discussed in section 4, in MC simulations, ICS and DCS are used to describe the energy and angular dependence of the scattering. On the other hand, a two-term solution expresses the DCS as an expansion in Legendre polynomials, where the zeroth order and first order coefficients of the expansion are related to the ICS and MT cross sections. The inclusion of anisotropic scattering in a two-term Boltzmann solver is further discussed in section 6 .

\subsection{On the validity of the Born approximation combined with the point-dipole interaction model}

A detailed theoretical analysis of the Born approximation combined with the point-dipole interaction can be found in the studies by Takayanagi [32], Crawford [31], and Shimamura and Takayanagi [33]. Here, we briefly summarize the main hypotheses underlying this approximation and their validity for calculating DCS of dipole rotational collisions of electrons in $\mathrm{CO}$, as compared with more advanced calculations methods. In particular, it is important to remember that the DCSs of equation (1) have been derived under the following main assumptions [32]:

(a) The interaction potential does not significantly alter the incident electron wave-function (weak potential approximation).

(b) The dipole part is the dominant contribution of the interaction and it can be represented by the point-dipole model, as described in [36].

(c) The first-order perturbation theory is appropriate to describe the collision processes.

The validity of assumptions (a) and (b) has been examined by Shimamura and Takayanagi [33] and by Takayanagi [32] by evaluating the relative importance of various partial cross sections. Their study points out that the Born approximation is not as poor as one might expect, even for low incident electron energies. This is due to the fact that the effective region of interaction is distant from the molecule. Therefore, the electron wave-function is only slightly distorted from its incident form. For this reason, the point-dipole interaction may be adopted, since only distant encounters are important. The same arguments hold also for quadrupole rotational collisions, as shown by Gerjuoy and Stein [37]. The validity of this approximation at low energies is confirmed also by theoretical studies by Crawford and Dalgarno [38], who applied the close-coupling method to the calculation of dipole rotational cross sections in CO, and by Jain and Norcross [39], who made ab initio calculations of the interaction of electrons and CO. These studies show that the electron-dipole interaction dominates in scattering at low energies, for $\epsilon<0.05 \mathrm{eV}$. Moreover, their ICSs are in good agreement with the ones reported by Itikawa and Mason [40], derived from the Born-dipole theory. For intermediate energies, typically around $1 \mathrm{eV}$, the first-order theory is no longer applicable to obtain reliable results. This is due to the fact that polarizability and short-range interactions can distort the incident electron wave-function. In these conditions, corrections to the Born approximation can be included via $R$ matrix [41] or close-coupling calculations [36]. For increasing values of energies (i.e. above $10 \mathrm{eV}$ ), collisions of electrons are often fast enough to make collision much shorter than the molecular rotation. Therefore, rotational cross sections are primarily determined by rotational constants, such as the dipole and quadrupole moment.

The validity of assumption (c) is related to the magnitude of the dipole moment of the $\mathrm{CO}$ molecule. In particular, since the dipole moment of $\mathrm{CO}$ is not large, a simple perturbation theory, such as the one based on the Born approximation, is applicable. In [42], it is discussed that the Born approximation provides a very poor estimate of the MT cross section for dipole rotational collisions, unless the dipole moment is small (i.e. $\mu<0.5 e a_{0}$ ).

To test the validity of DCSs derived from the Born-dipole treatment, accurate calculations are required, by assuming a more realistic interaction. An example of such calculations 


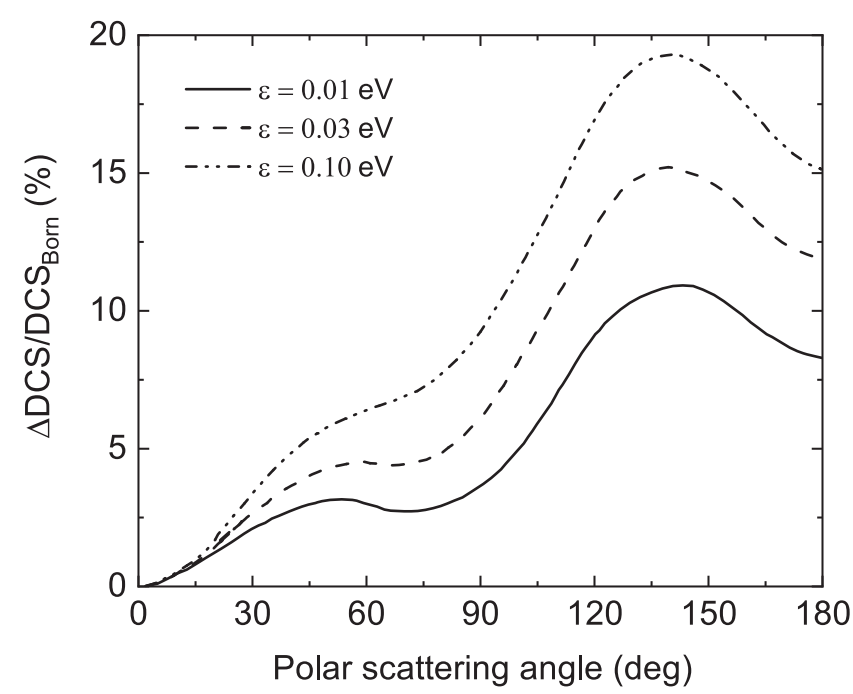

Figure 3. Percent deviations between DCSs for the $J=0 \rightarrow 1$ rotational excitation by electron impact in $\mathrm{CO}$ obtained from close-coupling calculations by Crawford and Dalgarno [38] and from Born-dipole theory (equation (1)), as a function of the polar scattering angle, for different incident electron energies, namely $0.01 \mathrm{eV}$ (solid line), $0.03 \mathrm{eV}$ (dashed line), and $0.1 \mathrm{eV}$ (dashed-dotted line).

are the ones performed by Crawford and Dalgarno [38], based on the close-coupling method, where dipole, quadrupole and short-range interactions in spherically symmetric form are included. Percent differences between DCS for the dipole transition $0 \rightarrow 1$ derived from the Born theory and close-coupling calculations by Crawford and Dalgarno are shown in figure 3. The percent differences are reported as $\triangle \mathrm{DCS} / \mathrm{DCS}_{\mathrm{Born}}(\%)=$ $\left(\mathrm{DCS}_{\mathrm{Born}}-\mathrm{DCS}_{\mathrm{CD}}\right) / \mathrm{DCS}_{\mathrm{Born}} \cdot 100$, where $\mathrm{DCS}_{\mathrm{CD}}$ is the expression of DCS as in [38], and $\mathrm{DCS}_{\mathrm{Born}}$ comes from equation (1). Three different incident electron energies are considered, namely $0.01,0.03$ and $0.1 \mathrm{eV}$.

Figure 3 shows that the agreement worsens when increasing the incident electron energy from $0.01 \mathrm{eV}$ to $0.1 \mathrm{eV}$. In particular DCSs obtained from the Born-dipole theory overestimate the contribution of backward scattering in dipole collisions, due to missing coefficients in equation (1) that include the dependence of the DCS on transition probability matrices with different angular momentum. Nevertheless, percent deviations between the results obtained from the two different approaches are within $20 \%$, when the incident electron energy is sufficiently small (i.e. $\epsilon \leqslant 0.1 \mathrm{eV}$ ). Similar conclusions for the $0 \rightarrow 1$ transition have also been drawn by Itikawa and Takayanagi [43] for other two polar molecules, HCI and $\mathrm{CN}$. Moreover, in [38], it is also shown that quadrupole interactions are almost isotropic. This description is coherent with the Born theory and the same assumption is employed in this work in sections 5.2 and 6 , where the addition of quadrupole collisions is investigated. To summarize, at low incident electron energies, the scattering is dominated by dipole rotational collisions of electrons with $\mathrm{CO}$ molecules. Moreover, since the scattering is distant and the $\mathrm{CO}$ dipole moment is not large, the first Born approximation combined with the pointdipole interaction model provides a simple and effective way to accurately describe the angular scattering component of electron-dipole and electron-quadrupole interactions in CO. Note that the same conclusions cannot be trivially extended to other polar molecules, such as $\mathrm{H}_{2} \mathrm{O}$, where the inclusion of short-range interactions is important not only to obtain accurate DCSs, but also for a correct estimation of ICSs of rotational collisions [41].

\section{Electron impact cross sections sets in CO}

Two different datasets of cross sections for electron impact in $\mathrm{CO}$ are considered. The first set was recently proposed by the Lisbon group [18] and it is available at the IST-Lisbon database of LXCat [19]. This set has been optimized from calculations of electron swarm parameters in $\mathrm{CO}$, using the two-term Boltzmann solver LoKI-B [20]. The second set was obtained from Biagi's MC simulations code Magboltz v11.10 [24]. Biagi's set is not available at LXCat, thus cross sections have been carefully transcribed from the source code into the LXCat format. To the best of our knowledge, there are no other independent works using this dataset, hence, this section is dedicated to its description.

The cross sections included in Magboltz v11.10 are shown in figure 4. These data were adjusted to reproduce measurements of electron swarm parameters from Haddad and Milloy [44], Petrović and Crompton [45], Nakamura [46], Saelee and Lucas [47], and Pack et al [48], for temperatures between $77 \mathrm{~K}$ and $443 \mathrm{~K}$. In particular, the set is composed by 1 elastic MT cross section for the ground electronic state (i.e. $\mathrm{CO}(X)$ ); 26 dipole rotational excitations of the ground vibrational state $\mathrm{CO}(X, \nu=0)$ (i.e. $\mathrm{CO}(X, \nu=0, J=0) \rightarrow \operatorname{CO}\left(X, \nu=0, J^{\prime}\right)$, with $\left.J^{\prime}=1-26\right)$; 6 vibrational excitations from $\mathrm{CO}(X, \nu=0)$; six electronic excitations of $\operatorname{CO}(X, \nu=0) ; 1$ ionization and 1 dissociative electron attachment to $\mathrm{O}^{-}$. Superelastic collisions from excited rotational and vibrational levels are also included in Magboltz, as they are essential for accurate calculations of electron swarm parameters at low and moderate $E / N$ values (i.e. $0.01 \leqslant E / N \leqslant 100 \mathrm{Td}$ ). Cross sections for these processes are calculated with the Klein-Rosseland formula (equation (10)) [35].

Electron scattering from $\mathrm{CO}$ has similarities with that of $\mathrm{N}_{2}$, leading to the observation that the transport properties are similar in both gases [1]. Like nitrogen, $\mathrm{CO}$ does not have a Ramsauer-Townsend minimum. Moreover, in both gases, the elastic MT cross section decreases at low electron energies (i.e. below $0.5 \mathrm{eV}$ ) exhibiting a pronounced resonance peak around $2 \mathrm{eV}$ and a negative slope for energies greater than $2 \mathrm{eV}$ [1]. The difference with respect to nitrogen is that $\mathrm{CO}$ is a heteronuclear molecule, with a permanent dipole moment $\mu=0.11 \mathrm{D}$ in the ground state, hence, interactions between electrons and the molecular dipole are dominant in the low energy region. In figure 4 , it can be seen that below $0.3 \mathrm{eV}$ the cross section for the rotational transition $J=0 \rightarrow 1$ is larger than the elastic MT. In Magboltz v11.10, electron impact rotational excitations are generated with the formulas of Takayanagi [32] for dipole rotational transitions (i.e. equations (3) and (4)). In total, Biagi has considered 26 electron impact rotational excitations/deexcitations from $J=0$ up to $J=26$, satisfying the selection 


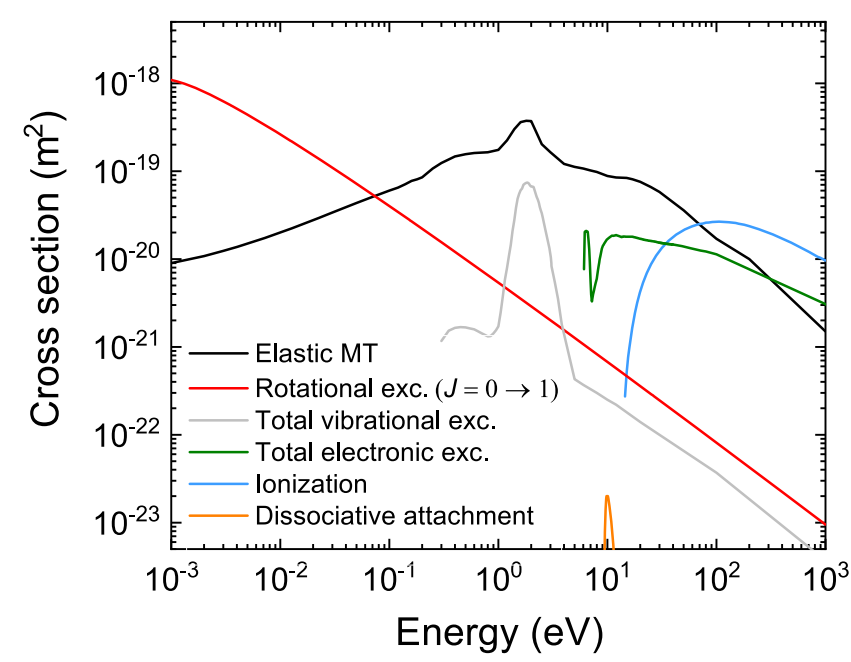

Figure 4. Biagi's electron impact cross sections for $\mathrm{CO}$, as a function of electron energy, obtained from the Magboltz code v11.10 [24]. Vibrational and rotational excitation cross sections are total cross sections, given by the sum of contributions from individual levels, where the fractional populations are calculated according to a Boltzmann distribution at $300 \mathrm{~K}$

rule $|\Delta J|=1$ for dipole processes, all exhibiting the typical trend of an allowed-dipole transition, with a decrease as $\log (\epsilon) / \epsilon$ at increasing energy, as given by equations (3) and (4).

Figure 5(a) highlights the differences between Biagi and IST-Lisbon cross sections for elastic MT and rotational excitations (here, only $\sigma_{0,1}^{\mathrm{ICS}}(\epsilon)$ is plotted as far as rotational collisions are concerned). Specifically, the elastic MT cross sections from the two datasets agree reasonably well for energies above $0.5 \mathrm{eV}$, whereas large discrepancies are present at lower energies. These discrepancies are related to the ICS for rotational excitations adopted in the two datasets, which, in turn, show large discrepancies for energies below $0.03 \mathrm{eV}$. In fact, Biagi calculates ICSs for rotational excitations from equations (3) and (4), whereas the rotational cross sections by IST-Lisbon are adjusted to have a match between experimental measurements and numerical results, obtained with the two-term solver LoKI-B [20], for the electron swarm parameters. Additionally, these adjusted rotational cross sections are subtracted from Phelps effective MT to obtain an elastic MT cross section. Note that it is hard to measure an elastic MT cross section in this case, due to the difficulties in separating the elastic contribution from the individual rotational transitions in an experimental energy loss spectrum [15]. Hence, on the one hand, the IST-Lisbon set is obtained assuming isotropic scattering, without any distinction between ICS and MT cross sections and it is adjusted to be used in a two-term Boltzmann solver. On the other hand, Biagi's set uses ICSs for rotational collisions (from equations (3) and (4)) in the Born approximation, in combination with an anisotropic scattering model to describe the transfer of momentum in MC simulations. It is clear that, in this case, the choice of the angular scattering model has an impact on the optimization of the cross sections set, based on swarm analysis. In fact, while the isotropic assumption implies that a single set of rotational cross sections is used to describe both energy and momentum exchanges, using an anisotropic model introduces differences between ICS and MT cross sections. A more detailed investigation on angular scattering models for dipole-rotational collisions in MC simulations codes is presented in sections 4 and 5 .

As regards higher energy threshold processes, six different cross sections for electron impact vibrational excitation of the ground vibrational state $\operatorname{CO}(X, \nu=0)$ are available in Biagi's set. Second-kind (superelastic) collisions from vibrational states of the ground electronic state are also included, assuming micro-reversibility [35]. These vibrational excitation cross sections are in good agreement with measurements by Gibson and co-authors [51], Ehrhardt and co-authors [52] and Land [14], and also with recent calculations by Laporta and co-authors [53]. Cross sections from [53] are also used in the IST-Lisbon dataset [18]. As opposed to the Lisbon dataset, Biagi's vibrational excitation cross sections are divided by a normalizing factor of 1.3, as suggested in the work by Haddad and Milloy [44]. Moreover, for energies above $4 \mathrm{eV}$, Biagi's cross sections are extrapolated linearly in a double logarithmic scale.

Excitations to higher vibrational states of $\mathrm{CO}(X)$ are also included in the IST-Lisbon set, up to $\nu=10$. However, the contribution of these additional processes in the calculation of electron transport parameters is expected to be negligible. In fact, under swarm conditions, the transition $\nu=0 \rightarrow 1$ is the most important one, due to the lower cross sections for transitions to higher levels and the small populations of levels above the $\nu=0$ one

Biagi's set includes electron impact excitations to the following electronic states: $\mathrm{CO}\left(a^{3} \Pi\right), \mathrm{CO}\left(a^{\prime 3} \Sigma^{+}\right), \mathrm{CO}\left(A^{1} \Pi\right)$, $\mathrm{CO}\left(b^{3} \Sigma^{+}\right), \mathrm{CO}\left(E^{1} \Pi+C^{1} \Sigma^{+}\right)$and $\mathrm{CO}(13.5 \mathrm{eV})$. The cross sections are the same as the ones used in swarm analysis by Land [14] and by Hake and Phelps [13], where the excitation to $\mathrm{CO}\left(E^{1} \Pi+C^{1} \Sigma^{+}\right)$is given by the sum of the corresponding individual cross sections. The 'state' denoted as $\mathrm{CO}(13.5 \mathrm{eV})$ is a composite of several states that present a broad peak in the electron impact excitation spectra, as suggested by Sawada and co-authors [50].

Total electronic excitation cross sections from Biagi [24], IST-Lisbon [18], Raju [1], Kanik and co-authors [49] and Sawada and co-authors [50] are plotted in figure 5(b). As expected, Biagi's total electronic excitation cross section is in good agreement with the one from Sawada and co-authors [50], since most of Biagi's cross sections are taken from this source. Note that Biagi's cross sections are linearly extrapolated in double logarithmic scale, for energies above $100 \mathrm{eV}$. The small peak in Biagi's cross section near $6.0 \mathrm{eV}$ is due to a resonance structure in the $a^{3} \Pi$ excitation cross section, as measured by Swanson and co-authors [54]. This peak is not present in the other cross sections sets, probably due to the negligible effect it has on the calculated electron transport parameters in pure CO. However, as suggested by Haddad and Milloy [44], omitting the peak in the $a^{3} \Pi$ excitation cross section gives a difference of about $18 \%$ between calculated and measured values of electron drift velocities, for a mixture of $1 \% \mathrm{CO}$ in argon. 

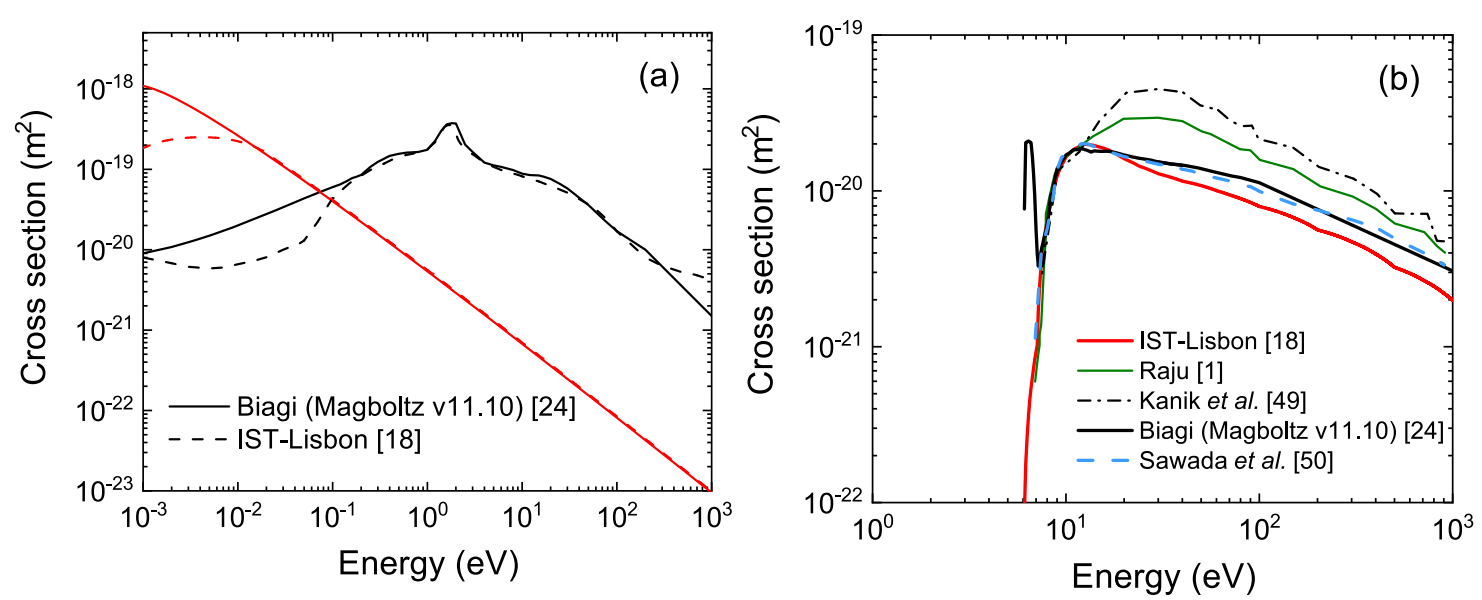

Figure 5. (a) Elastic momentum transfer cross sections of electrons in $\mathrm{CO}(X)$ (black lines) and electron impact rotational excitation $\mathrm{CO}(X, \nu=0, J=0) \rightarrow \mathrm{CO}\left(X, \nu=0, J^{\prime}=1\right)$ (red lines), from IST-Lisbon (dashed lines) [18] and Biagi (solid lines) [24] datasets, and (b) total electronic excitation cross sections from $\operatorname{CO}(X, \nu=0)$, from different sources: Raju [1] (green solid line), IST-Lisbon [18] (red solid line), Biagi [24] (black solid line), Kanik et al [49] (dashed-dotted line), and Sawada et al [50].

Differences between Biagi's and IST-Lisbon total electronic excitation cross sections are due to the fact that, in the IST-Lisbon dataset [18], cross sections for excitation to $a^{3} \Pi$ and $b^{3} \Sigma^{+}$are taken from Sawada and co-authors [50], whereas for all other electronic excitations are taken from Itikawa [15]. Moreover, differences with the set of Kanik and co-authors [49] are due to the fact that the latter considers the contribution of $a^{3} \Pi, a^{3} \Sigma^{+}, d^{3} \Delta$ and $A^{1} \Pi$ only, increased by $20 \%$. The cross section recommended in the book by Raju [1] is an average between the one proposed by Sawada and co-authors [50] and the one recommended by Kanik and co-authors [49]. It should be noted that the book by Raju [1] was published after the 2003 update of Biagi's cross sections. Recently, a new set of electron impact cross sections has been released in Magboltz v11.11 [55]. The update includes (i) anisotropic scattering for dipole rotational collisions based on the angular scattering model by Okhrimovskyy and co-authors [56], (ii) 11 electron impact vibrational excitations of the ground vibrational state taken from the work by Allan [57], (iii) measured oscillator strengths for dipole transitions, and (iv) separated contributions from ionization and eight dissociative ionization channels. The recent changes related to the anisotropic scattering of rotational collisions are further discussed in the next section. A detailed comparison between measured and calculated electron swarm parameters, using the 2021 version of Biagi's electron impact cross sections, will be investigated in details in the future. Since the agreement obtained with the current set [24] is already very good, we expect that the main results presented in this work still apply for the 2021 set.

\section{Angular scattering models for electron-neutral rotational collisions in Monte Carlo simulations}

In order to compare different angular scattering models, it is useful to define the angular distribution function $I(\epsilon, \theta)$, by normalizing the DCS as

$$
I(\epsilon, \theta)=\frac{1}{\sigma^{\operatorname{ICS}}(\epsilon)} \frac{\mathrm{d} \sigma(\epsilon, \theta)}{\mathrm{d} \Omega} .
$$

The advantage of using this formulation is that one might keep the same ICS, while varying the angular scattering model $[27,58]$. The considered angular distributions are based on isotropic scattering, empirical formulas given by Longo and Capitelli [59] and Kushner [29], and the dipole-Born theory [31]. The scattering angles in electron-molecule collisions can be sampled according to a theorem of probability theory [60], by inverting the following expression:

$$
r_{i}=2 \pi \int_{0}^{\theta_{i}} I\left(\epsilon, \theta^{\prime}\right) \sin \theta^{\prime} \mathrm{d} \theta^{\prime},
$$

where $r_{i}$ is a collection of random numbers uniformly distributed between 0 and $1, \theta_{i}$ is a collection of random numbers distributed according to a probability distribution function $p\left(\epsilon, \theta^{\prime}\right)=2 \pi I\left(\epsilon, \theta^{\prime}\right) \sin \theta^{\prime}$ in $[0, \pi]$ and $p\left(\epsilon, \theta^{\prime}\right)$ is normalized to 1 in $[0, \pi]$.

The angular distribution for isotropic scattering is constant, i.e.

$$
I(\epsilon, \theta)=\frac{1}{4 \pi}, \quad \cos \theta_{i}=1-2 r_{i} .
$$

Moreover, this scattering model leads to equal ICS and MT cross sections (equations (2) and (5)), in contrast with a description of dipole rotational collisions based on the Born approximation, as described in the works by Crawford [31] and by Shimamura and Takayanagi [33].

Longo and Capitelli [59] have introduced an anisotropic scattering model that takes into account the differences between ICSs and MT cross sections. The model is based on the definition of an artificial DCS that satisfies equations (2) and (5). The artificial DCS is composed by the sum of two constant DCSs, one for isotropic forward scattering (i.e. $0 \leqslant$ $\theta \leqslant \pi / 2$ ) and the other one for isotropic backward scattering 


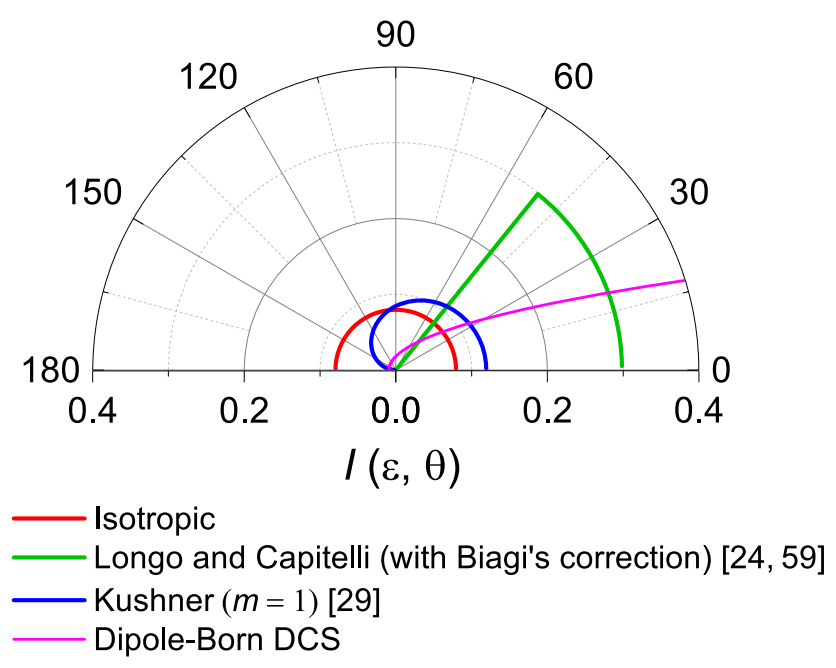

Figure 6. Polar plot of the angular distribution functions $I(\epsilon, \theta)$ at $\epsilon=0.01 \mathrm{eV}$ for the isotropic scattering model (red line) and the anisotropic scattering models by Longo and Capitelli with Biagi's correction [24, 59] (green line), Kushner [29] (blue line) and the dipole-Born DCS proposed in this work (magenta line).

(i.e. $\pi / 2<\theta \leqslant \pi$ ). With these assumptions, the forward scattering probability can be calculated as:

$$
P_{\mathrm{F}}(\epsilon)=\frac{1}{2}+\gamma(\epsilon), \quad \gamma(\epsilon)=\frac{\sigma^{\mathrm{ICS}}(\epsilon)-\sigma^{\mathrm{MT}}(\epsilon)}{\sigma^{\mathrm{ICS}}(\epsilon)},
$$

where the term $\gamma(\epsilon)$ accounts for the relative difference between ICS and MT cross sections. A strongly forwardpeaked model for rotational collisions (i.e. $P_{\mathrm{F}}(\epsilon)>0.5$, for any $\epsilon$ ) is obtained, by using equations (3) and (6) in equation (18). This model was implemented in Magboltz v11.10 [24] with a renormalization of the forward scattering probability according to

$$
P_{\mathrm{F}}(\epsilon)=\frac{1}{2}+\gamma(\epsilon) \frac{\left(1-\cos \Theta_{m}\right)}{\sin ^{2} \Theta_{m}},
$$

where $\Theta_{\mathrm{m}}=\sin ^{-1}(2 \sqrt{\gamma(\epsilon)(1-\gamma(\epsilon))})$ defines the maximum polar scattering angle (which is found around $60^{\circ}$, in this case). As a consequence, the polar scattering angle $\theta_{i}$ is sampled according to isotropic scattering between 0 and $\Theta_{\mathrm{m}}$ :

$$
\cos \theta_{i}=1-\alpha r_{i}
$$

where $\alpha=2 \Theta_{\mathrm{m}} / \pi$. In [29], Kushner proposes an empirical expression for the angular distribution of the form:

$$
I(\epsilon, \theta)=\frac{m+2}{8 \pi} \cos ^{m}(\theta / 2),
$$

where $m$ is an energy dependent fitting parameter used to reproduce experimental DCSs. If experimental measurements of DCSs are not available, $m$ is assumed constant. In [28], Casey and co-authors have considered $m=1$, to describe the anisotropy of electron rotational collisions in $\mathrm{N}_{2}$. In this case, the scattering angle in the polar direction can be calculated from equation (16) as

$$
\theta_{i}=2 \cos ^{-1}\left[\left(1-r_{i}\right)^{1 / 3}\right] .
$$

Note that equation (21) assumes that $I(\epsilon, \theta)$ is independent on the kinetic energy of the incident electrons. Typically, this assumption is valid in the region of resonant vibrational excitations [52], but it is not able to reproduce the DCS over a large energy spectrum. The dependence of $I(\epsilon, \theta)$ on both kinetic energy of electrons and scattering angle is investigated in the next subsection.

\subsection{Anisotropic scattering model from the dipole-Born differential cross sections}

In this subsection, a novel anisotropic scattering model for the description of dipole-rotational collisions in MC simulations is derived. The model aims to overcome the inconsistencies and/or limitations previously described and it is based on the Born theory illustrated in section 2. The model departs from the coherent expressions for the differential and ICSs for rotational transitions, given by equations (1) and (15), respectively. By substitution of equations (1) and (3) into equation (15), the following angular distribution function $I(\epsilon, \theta)$ is obtained:

$$
\begin{aligned}
I(\epsilon, \theta)= & \frac{1}{4 \pi} \frac{\sqrt{\epsilon} \sqrt{\epsilon \pm V_{J, J \pm 1}}}{K^{2}} \\
& \times\left[\log \left(\frac{\sqrt{\epsilon \pm V_{J, J \pm 1}}+\sqrt{\epsilon}}{\sqrt{V_{J, J \pm 1}}}\right)\right]^{-1} .
\end{aligned}
$$

Hence, by substitution of equation (23) in equation (16) and integration, an expression for the polar scattering angle $\theta_{i}$ is derived for use in MC simulations codes:

$$
\cos \theta_{i}=1+\frac{2 \xi^{2}}{1-\xi^{2}}\left(1-\xi^{-2 r_{i}}\right),
$$

where $\xi$ is an energy-dependent function of the form:

$$
\xi(\epsilon)=\frac{V_{J, J \pm 1}}{\left(\sqrt{\epsilon \pm V_{J, J \pm 1}}+\sqrt{\epsilon}\right)^{2}} .
$$

Since the energy exchange in rotational transitions is typically found in the range $10^{-4}-10^{-3} \mathrm{eV}$, it can be concluded that $\xi \ll 1$ and then equation (24) can be simplified as

$$
\cos \theta_{i} \sim 1-2 \xi^{2\left(1-r_{i}\right)} .
$$

Equation (26) corresponds to a strongly forward-peaked scattering model with values of $\cos \theta_{i}$ often very close to 1 . Nevertheless, scattering at extreme angles is still possible, for example at $r_{i}=1$.

A comparison between different angular distribution functions, obtained from the aforementioned models, is shown in figure 6. An incident electron energy of $\epsilon=0.01 \mathrm{eV}$ is assumed for the models of Longo and Capitelli and dipoleBorn DCS. For the latter, the transition $J=0 \rightarrow 1$ has been considered.

Kushner's angular distribution is almost isotropic, with a slight anisotropy in the forward direction, due to the fact that $m=1$ was chosen. From figure 6, it is clear that an artificial DCS is used in Longo and Capitelli's model that presents a more pronounced forward peak structure, when compared with Kushner's, due to the correction by Biagi that reduces 

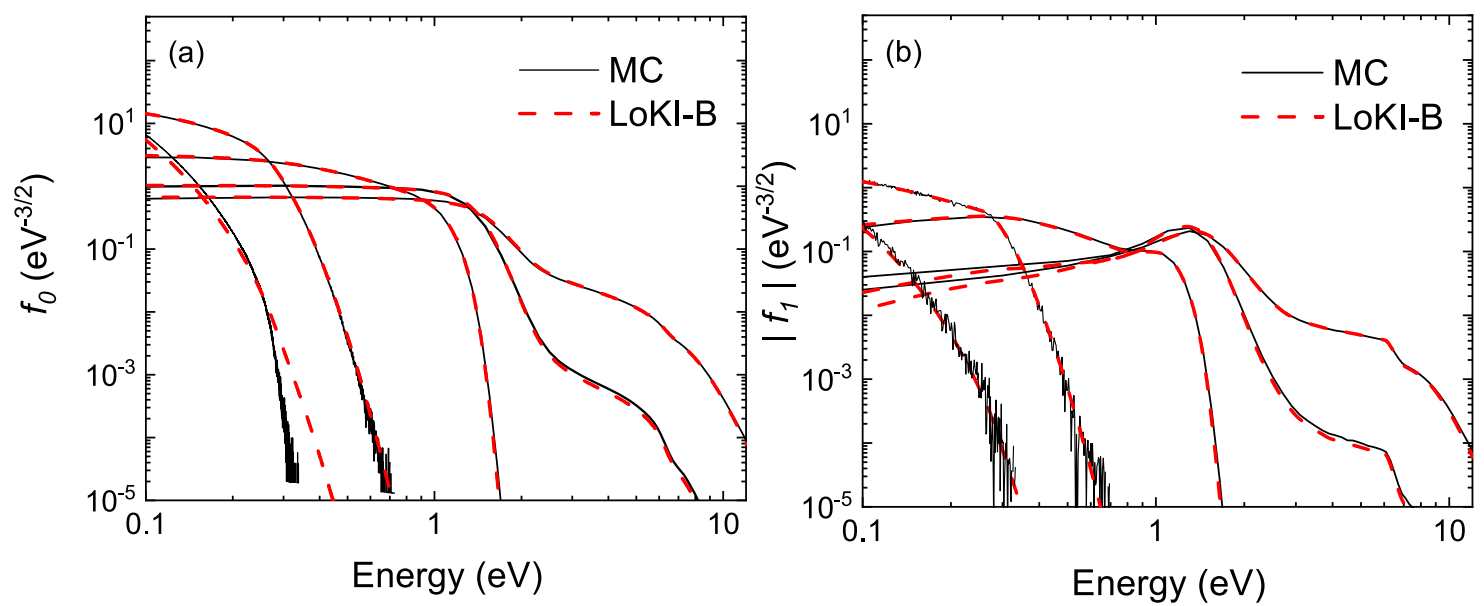

Figure 7. Zeroth (a) and first order (b) Legendre polynomial coefficients calculated with MC simulations (solid line) and LoKI-B [20] (dashed line) at different reduced electric fields (from left to right: 0.01, 1, 10, 50 and $100 \mathrm{Td}$ ), using Biagi's cross sections [24]. A Boltzmann distribution at $300 \mathrm{~K}$ for rotational and vibrational levels of the ground electronic state is assumed.

the forward scattering component to maximum polar angles $\Theta_{\mathrm{m}}<60^{\circ}$. Scattering angles above $\Theta_{\mathrm{m}}$ are not allowed by the artificial shape of the DCS. The angular scattering model based on the dipole-Born DCS proposed here differs significantly from the other three. This model includes not only a dependence on the polar scattering angle, thus improving the scattering description, but also considers a dependence on the electron energy before and after the collision, thus overcoming the limitations of the empirical formulas by Kushner. Moreover, in the proposed model, the scattering angle is sampled directly from the dipole-Born DCS, instead of an artificial DCS and a forward scattering probability. Nevertheless, it should be emphasized that the dipole-Born anisotropic model is valid only for this specific type of transitions, whereas the other three models are generally applicable to other types of scattering.

\section{Calculations of electron swarm parameters in CO}

In this section, we aim to assess the validity of the two-term approximation for electrons in CO. Moreover, we verify the effects of different anisotropic scattering models for dipole rotational collisions on the calculations of electron swarm parameters. Anisotropic effects in vibrational and electronic excitations were not considered, since the comparison of the calculated and measured swarm data is already very good for $E / N>1 \mathrm{Td}$, where these processes are dominant, with the current cross section sets. Nevertheless, the study of these effects is interesting and can provide valuable insight for future improvements of the cross section sets.

A fully native MC simulations code is used for simulations of the electron kinetics in $\mathrm{CO}$ and calculations of electron transport parameters. The code was previously benchmarked against solutions of two-term and multi-term Boltzmann solvers and was combined with variance reduction techniques in atomic and molecular gases simulations [22, 23]. It uses a null-collision method [61], along with a modified time-step technique [62] and it includes the effects of the finite temperature of the background gas with an exact test particle $\mathrm{MC}$ technique that takes into account the translational distribution of neutrals [63]. According to this treatment, the frequency for an arbitrary electron-neutral collision is

$$
\nu(v)=N \int_{\boldsymbol{u}^{\prime}} \sigma(|\boldsymbol{v}-\boldsymbol{u}|)|\boldsymbol{v}-\boldsymbol{u}| F(\boldsymbol{u}) \mathrm{d} \boldsymbol{u},
$$

with $N$ the number gas density, $\boldsymbol{v}$ the velocity of the incident electron, $\boldsymbol{u}$ the velocity of the background gas, $\sigma(|\boldsymbol{v}-\boldsymbol{u}|)$ the corresponding electron-impact cross section, and $F(\boldsymbol{u})$ the velocity distribution function of the background gas particles, normalized as

$$
\int_{\boldsymbol{u}^{\prime}} F(\boldsymbol{u}) \mathrm{d} \boldsymbol{u}=1
$$

In this work, a Maxwell-Boltzmann distribution for the background gas particles is used, motivated by the swarm conditions under investigation. The same approach has been adopted by Vialetto and co-authors [23] for calculations of electron transport parameters in $\mathrm{CO}_{2}$. Details about the method can be found in [63]. The scattering event is simulated in the centreof-mass frame, requiring cross sections written in the same reference frame. Formulas for converting cross sections from the laboratory to the centre-of-mass frame can be found in [64]. Here, the differences between cross sections in the center-ofmass and in the laboratory frame are negligible, due to the low electron-to-neutral mass ratio. After each scattering event, the magnitude of the electron velocity vector is updated based on energy conservation and the velocity components are rotated based on trigonometric expressions [65]. In [23], the MC Flux method is used for calculations of flux transport coefficients, whereas here, a standard time-of-flight (TOF) MC method for the calculation of bulk transport parameters [66] is employed. Hence, the trajectories of a large number of electrons (typically $10^{4}$ or $10^{5}$ ) are followed in swarm conditions, under the effects of collisions with the background gas and of a constant DC electric field. To ensure that steady-state is reached, the 
percent deviation of the mean kinetic energy at every timestep is calculated. The steady-state time $t_{\mathrm{SS}}$ is defined as the time when this deviation is below $0.1 \%$. Once $t_{\mathrm{SS}}$ is reached, results for bulk transport parameters are averaged with a sample time of $10^{-9} \mathrm{~s}$ until a final time $t_{\text {fin }}=10 t_{\mathrm{SS}}$, in order to improve statistics. In addition to the MC simulations code, we have also used the Boltzmann solver LoKI-B [20, 67], based on a two-term expansion of the EVDF in Legendre polynomials and adopting a finite difference scheme to discretise the electron Boltzmann equation in energy space. As usual in two-term Boltzmann solvers, LoKI-B assumes isotropic scattering of electrons in rotational collisions. In section 6 , this assumption is dropped and the treatment of dipole rotational collisions in LoKI-B is extended to include anisotropic effects. It is important to mention that LoKI-B provides calculations of flux transport parameters, whereas MC simulations calculate bulk transport parameters [12] from the spatio-temporal evolution of the electron swarm, according to a standard TOF formulation $[66,68]$. However, for low/moderate values of $E / N$ (i.e. below $100 \mathrm{Td}$ ) in $\mathrm{CO}$, the differences between flux and bulk coefficients are below $1 \%$, meaning that the general considerations of this study are not affected by these different formulations. Indeed, distinction between flux and bulk parameters in $\mathrm{CO}$ must be taken into account at higher $E / N$ values, where the role of non-conservative inelastic collisions is dominant [69].

In order to compare MC simulations results with the ones obtained with LoKI-B, we start by considering isotropic scattering of electrons in rotational collisions. The calculations are performed for $E / N$ values between 0.01 and $100 \mathrm{Td}$, using the cross sections set of Biagi. In MC simulations, the trajectories of $10^{4}$ initial electrons are followed. An energy bin size of $10^{-4} \mathrm{eV}$ for $E / N \leqslant 10 \mathrm{Td}$ and of $10^{-1} \mathrm{eV}$ for $E / N>10 \mathrm{Td}$ is considered. The calculations with the solver LoKI-B are performed for the same $E / N$ values, assuming a temporal growth of the electron density and adopting 1000 energy cells that are automatically adjusted by prescribing the decade-fall of the electron energy distribution function between 20 and 25 [20]. In both MC simulations and LoKI-B calculations, we consider a Boltzmann population at $300 \mathrm{~K}$ for the vibrational and the rotational levels of the electronic ground state, where vibrational energies are calculated using the Morse anharmonic oscillator model and rotational energies are calculated with a rigid rotor model [70]. Figures 7(a) and (b) show the zeroth $\left(f_{0}\right)$ and the first order $\left(f_{1}\right)$ Legendre polynomial coefficients, respectively, calculated from LoKI-B and MC simulations. Normalization is such that

$$
\int_{0}^{\infty} f_{0}(\epsilon) \sqrt{\epsilon} \mathrm{d} \epsilon=1,
$$

with $\epsilon$ the electron kinetic energy. The CPU time for a single calculation is around $10 \mathrm{~s}$ for LoKI-B and up to $1 \mathrm{~h}$ for $\mathrm{MC}$ simulations, due to the long time for averages required by the simulations, obtained with a $2.6 \mathrm{GHz}$ six-Core Intel Core 17 processor. Figure 7 reveals a relatively good agreement between MC simulations and LoKI-B results, with small deviations observed only in the body and in the tail of $f_{0}$ and $f_{1}$. Similar conclusions are obtained when using the IST-Lisbon cross sections set [18] for the same range of $E / N$ (not shown).
As mentioned in section 3, there are similarities in the electron scattering cross sections of $\mathrm{CO}$ and $\mathrm{N}_{2}$, and thus also in the corresponding transport properties [1]. However, $\mathrm{N}_{2}$ yields significant deviations between two-term and multi-term/MC solutions, already at moderate values of $E / N$ [71], whereas calculations in this work confirm the validity of the two-term approximation in $\mathrm{CO}$. This fact is even more counter-intuitive if it is taken into account that the magnitude of inelastic cross sections for vibrational excitations of $\mathrm{CO}$ is about $30 \%$ greater than that of $\mathrm{N}_{2}$, in the same energy region [1]. This observation can be explained by noting that $\mathrm{CO}$, contrarily to $\mathrm{N}_{2}$, has a permanent dipole moment. In fact, rotational excitations by electron impact are driven by the interaction of electrons with the weak electric field resulting from the dipole moment of the CO molecules. In view of their very low energy thresholds, rotational processes contribute to the isotropization of electrons velocity, thus favouring the validity of the two-term approximation. In the case of $\mathrm{N}_{2}$, dipole rotational excitations do not naturally occur, hence rotational processes are of the electric quadrupole type [37], with cross sections typically one order of magnitude lower. Quadrupole rotational collisions are usually neglected in studies of electron kinetics in $\mathrm{CO}$, but, for completeness, the effect of these processes on the calculations of transport parameters is discussed in section 5.2.

\subsection{Assessment of anisotropy effects on swarm parameters using Monte Carlo simulations}

In order to test the assumption of isotropic scattering, calculations of electron transport parameters obtained with LoKI-B and MC simulations are compared with experimental measurements from the Dutton [72] and the LAPLACE [73] databases of LXCat. In addition to isotropic scattering, MC simulations have been performed taking into account also the three anisotropic scattering models for dipole rotational collisions described in section 4, using elastic MT and total inelastic cross sections from a single database. It should be noted that this assumption does not fix the total MT cross section [12], which changes when varying the angular distribution model associated with the dipole rotational collisions, thus inducing modifications in the calculated swarm parameters.

In figures 8(a) and (b), measurements and calculations of the electron reduced mobility $(\mu N)$ are compared in the range $0.01 \leqslant E / N \leqslant 100 \mathrm{Td}$, for gas temperatures $\left(T_{\text {gas }}\right)$ of $77 \mathrm{~K}$ and $300 \mathrm{~K}$, respectively. The results were obtained adopting the cross sections of Biagi [24] and the anisotropic scattering models presented in section 4. As for previous calculations, a Boltzmann distribution at $T_{\text {gas }}$ for rotationally and vibrationally excited states of the ground electronic state of $\mathrm{CO}$ molecules is assumed.

MC simulations assuming isotropic scattering are in excellent agreement with calculations from LoKI-B, within $2 \%$ in the $E / N$ range considered. The calculations are also in general good agreement with the measurements [72, 73] for $E / N>3$ Td. However, significant differences can be observed between measured and calculated values of $\mu N$ at lower $E / N$, for MC simulations with isotropic scattering and LoKI-B. In particular, at $77 \mathrm{~K}$, these differences exceed $50 \%$ (figure 8(a)), while at $300 \mathrm{~K}$ the differences are about $30 \%$ (figure $8(\mathrm{~b})$ ). 

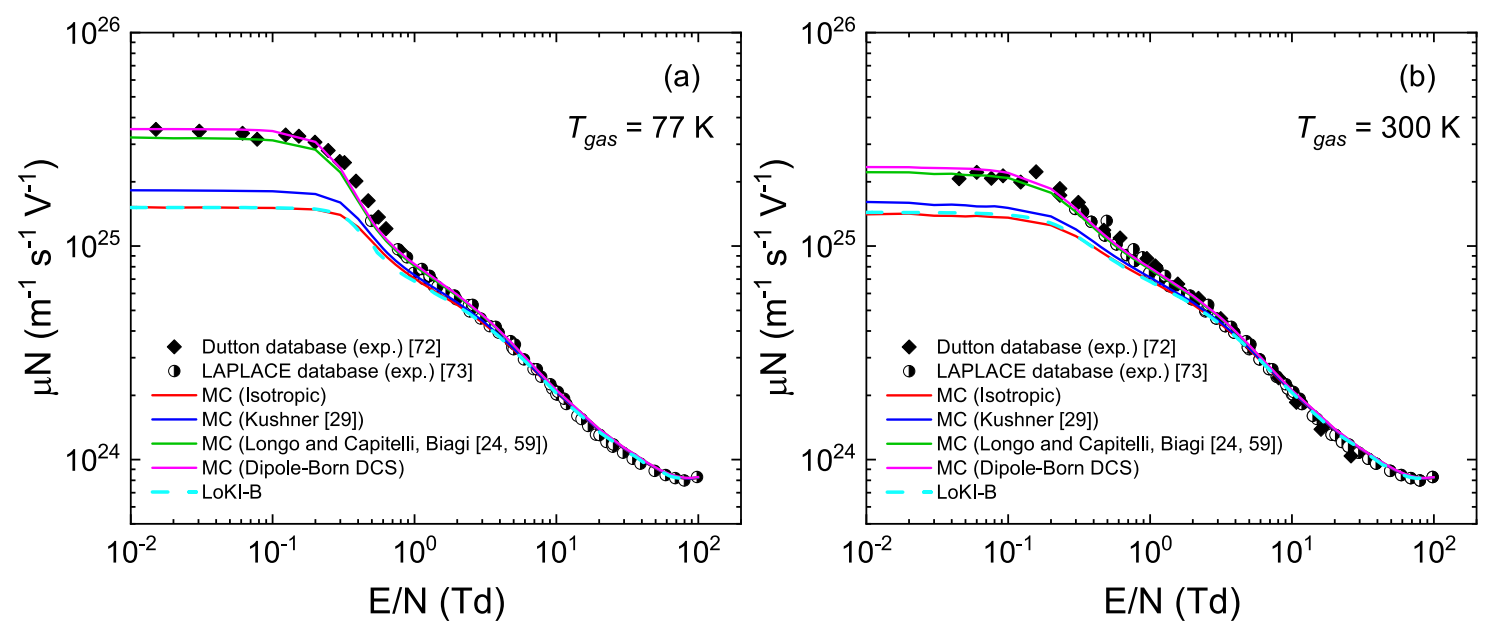

Figure 8. Reduced mobilities at $77 \mathrm{~K}$ (a) and $300 \mathrm{~K}$ (b) measured [72, 73] (points) and calculated from LoKI-B [20] (dashed curve) and MC simulations (solid curves), using the Biagi cross sections set and different angular scattering models for dipole rotational collisions. MC isotropic (red curve), MC with Kushner's model [29] (blue curve), MC with Longo and Capitelli's model with Biagi's correction [24, 59] (green curve) and MC with dipole-Born DCS model (magenta curve). A Boltzmann distribution at $T_{\text {gas }}$ for rotational and vibrational levels of the ground electronic state of $\mathrm{CO}$ is assumed.

When anisotropic scattering in rotational excitations and deexcitations is included in MC simulations, calculations of $\mu N$ are significantly affected. The effect is more evident for $E / N<3 \mathrm{Td}$, where the exchange of momentum is controlled by rotational processes. Specifically, MC simulations using the forward scattering model of Kushner with $m=1$ [29] lead to differences up to $17 \%$ at $77 \mathrm{~K}$ and up to $10 \%$ at $300 \mathrm{~K}$, when compared with the results of the isotropic model. These results are in agreement with the ones obtained by Casey and co-authors [28], where the same angular distribution function is used for the description of quadrupole rotational collisions in $\mathrm{N}_{2}$. Since the angular distribution of quadrupole interactions is isotropic, it is adequately described by Kushner's model, with $m=1$, that leads to an angular distribution close to the isotropic case. However, this also explains why this anisotropic model does not completely solve the discrepancies between experimental measurements and MC simulations when using the dipole rotational cross sections of Biagi. The anisotropic models of Longo and Capitelli [59] and dipole-Born DCS (section 4.1) for rotational processes lead to good agreement between MC simulations and measurements. In particular, at $300 \mathrm{~K}$, the calculated reduced mobilities agree with the experimental data within $2 \%$. Similar deviations can be found between measurements and calculations at $77 \mathrm{~K}$ for $E / N<0.2 \mathrm{Td}$ and $E / N>0.8 \mathrm{Td}$, but differences increase up to $8 \%$ for $0.2 \leqslant E / N \leqslant 0.8 \mathrm{Td}$, where $\mu N$ starts decreasing with $E / N$. As discussed in section 5.2, these discrepancies can be reduced by including quadrupole rotational excitations and de-excitations, that are neglected in calculations shown in figure 8 .

Although the most recent version of cross sections in Magboltz v11.11 [55] is not used in here, the general good agreement obtained with the model of Longo and Capitelli is expected, because Biagi's cross sections were adjusted for such agreement using this anisotropic description in Magboltz v11.10 [24]. However, that model is based on an artificial
DCS, not compatible with a Born formulation [33]. Moreover, the original model in [59] needs to be corrected due to the strongly forward-peaked nature of the scattering, as discussed in section 4. Alternatively, dipole-Born DCS can be used to calculate the polar scattering angle in rotational collisions. This description can be implemented in MC codes to simulate electron impact rotational collisions with linear molecules, in combination with ICSs described with the Born approximation (equation (3)).

Calculations for $\mu N$ were performed also with the ISTLisbon cross sections set [18]. MC simulations and LoKI-B results are shown in figures 9 (a) and (b), together with measurements [72, 73] obtained at $77 \mathrm{~K}$ and $300 \mathrm{~K}$, respectively.

Once again, significant differences among calculated reduced mobilities can be observed for $E / N<3 \mathrm{Td}$, when different angular scattering models are used in MC simulations. In particular, deviations exceeding 50\% are found between results from MC simulations with isotropic scattering and with a dipole-Born model for rotational processes at $77 \mathrm{~K}$, these differences decreasing to about $30 \%$ at $300 \mathrm{~K}$. However, contrarily to what is observed for results obtained with Biagi's cross sections, MC simulations assuming Kushner's anisotropic model with $m=1$ [29] lead to the best agreement with measurements. The use of other scattering models, like the one by Longo and Capitelli [59] or the dipoleBorn DCS, leads to strong overestimations of $\mu N$ for $E / N<$ $3 \mathrm{Td}$, when compared with experimental data. Nevertheless, since the IST-Lisbon cross sections set [18] was adjusted with the assumption of isotropic scattering, the apparent good agreement between experiments and MC simulations using Kushner's model is seen as fortuitous. On the other hand, deviations up to $20 \%$ can be found when using the isotropic model at $77 \mathrm{~K}$ in MC simulations or in LoKI-B, meaning that the net momentum exchange in rotational processes is not well captured by the IST-Lisbon cross sections set. As mentioned in section 4 , this is due to the fact that the isotropic assumption 

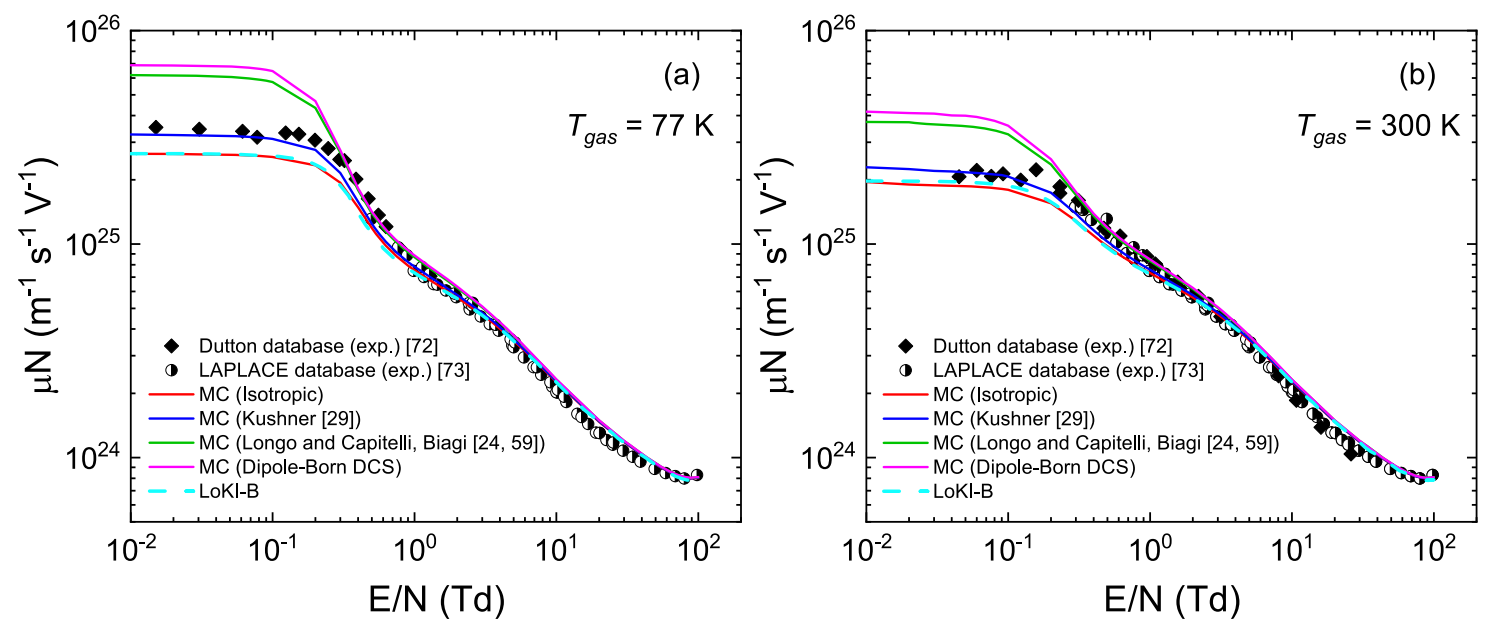

Figure 9. As in figure 8, but for calculations using the IST-Lisbon cross sections set [18].

leads to equal MT cross sections and ICSs (i.e. $\sigma^{\mathrm{MT}}=\sigma^{\mathrm{ICS}}$ ), which is inconsistent with the theoretical studies by Altshuler [21] and Crawford [31] on the MT theory for dipole rotational collisions. Moreover, this formulation may lead to negative values for the elastic MT cross section, if this is calculated from the total MT by Hake and Phelps [13] or Land [14], by subtracting the inelastic contribution [18].

For completeness, figures 10(a) and (b) show measurements [72] and calculations of the characteristic energy $\left(D_{\mathrm{T}} / \mu\right)$ at $77 \mathrm{~K}$ and $300 \mathrm{~K}$, respectively. The numerical results were obtained with LoKI-B and MC simulations, using Biagi's cross sections. Note that the values of $D_{\mathrm{T}} / \mu$ decrease with $E / N$, until the characteristic energy approaches $k_{\mathrm{B}} T_{\text {gas }}$, in the near thermal region.

A very good agreement is found between measurements and calculations, using both LoKI-B and MC simulations, with excellent numerical predictions (within $1 \%$ ) obtained at $300 \mathrm{~K}$ for $0.3 \leqslant E / N \leqslant 100 \mathrm{Td}$, where experimental data are available (see figure $10(\mathrm{~b})$ ). At $77 \mathrm{~K}$ and $0.01 \leqslant E / N \leqslant 0.3 \mathrm{Td}$, discrepancies between calculations and measurements up to $10 \%$ are found. The inclusion of anisotropic scattering leads to a significant improvement in the agreement for $0.06 \leqslant$ $E / N \leqslant 0.3 \mathrm{Td}$, whereas discrepancies can still be found for values between 0.01 and $0.06 \mathrm{Td}$. In [18], similar discrepancies are found in the same $E / N$ region using LoKI-B with the IST-Lisbon cross sections. As suggested by Hake and Phelps [13], these discrepancies can be due to the fact that experimental data are overestimated in the low $E / N$ region. Contrarily to what is observed for $\mu N$ calculations, that are strongly dependent on the angular scattering model, MC simulations for $D_{\mathrm{T}} / \mu$ are not significantly affected by the choice of the angular distribution in rotational collisions. This result can be explained by the fact that, as a first approximation, both the transversal diffusion coefficient $D_{\mathrm{T}}$ and the mobility $\mu$ are inversely proportional to the total MT cross section, their ratio becoming less sensitive to variations in this cross section. A more accurate explanation is given in section 6, where anisotropic effects in solutions of the two-term electron Boltzmann equation are considered.
In figure 11, the longitudinal component of the bulk diffusion tensor divided by the mobility $\left(D_{\mathrm{L}} / \mu\right)$, obtained from MC simulations, is compared with measurements [72], for $T_{\text {gas }}=300 \mathrm{~K}$. For this transport coefficient, measurements at $77 \mathrm{~K}$ are not available. As before, calculations were performed using Biagi's cross sections, assuming different angular scattering models for rotational collisions. With LoKI-B it is not possible to calculate the bulk longitudinal diffusion coefficient $D_{\mathrm{L}}$. As for $D_{\mathrm{T}} / \mu$, an excellent agreement is observed between calculations and measurements for all the angular scattering models considered here.

From the results of this section, it is clear that the choice of the angular scattering model for dipole rotational collisions has an impact on the comparison of calculated and measured swarm parameters larger than the choice of a two-term or an MC solution at steady-state. This is due to the very different trend of the ICS and MT cross sections (equations (3) and (6)), as shown in figure 2 . In fact, changing the angular distribution while keeping the sum of the ICSs unchanged, will affect the total MT cross section, hence transport parameters, such as the reduced mobility.

\subsection{Effect of quadrupole rotational collisions}

In this section, the effect of the inclusion of quadrupole rotational processes to Biagi's dataset is investigated. These processes are neglected in most of the available cross sections sets for $\mathrm{CO}$, due to the weakness of the quadrupole interaction. However, these interactions are not necessarily negligible and, depending on the molecular orientation relative to the velocity of the incident electron, quadrupole rotational transitions can occur [33]. The inclusion of rotational collisions was already suggested in the analysis by Hake and Phelps [13] and by Randell and co-authors [9]. Quadrupole excitations for CO have also been included in the work by Land [14], in the continuous approximation.

For very low electron energies (i.e. below the threshold of electronic excitation processes), the treatment of Gerjuoy and Stein [37] for electric quadrupole transitions is justified. They deduce an effective cross section, based on the Born 

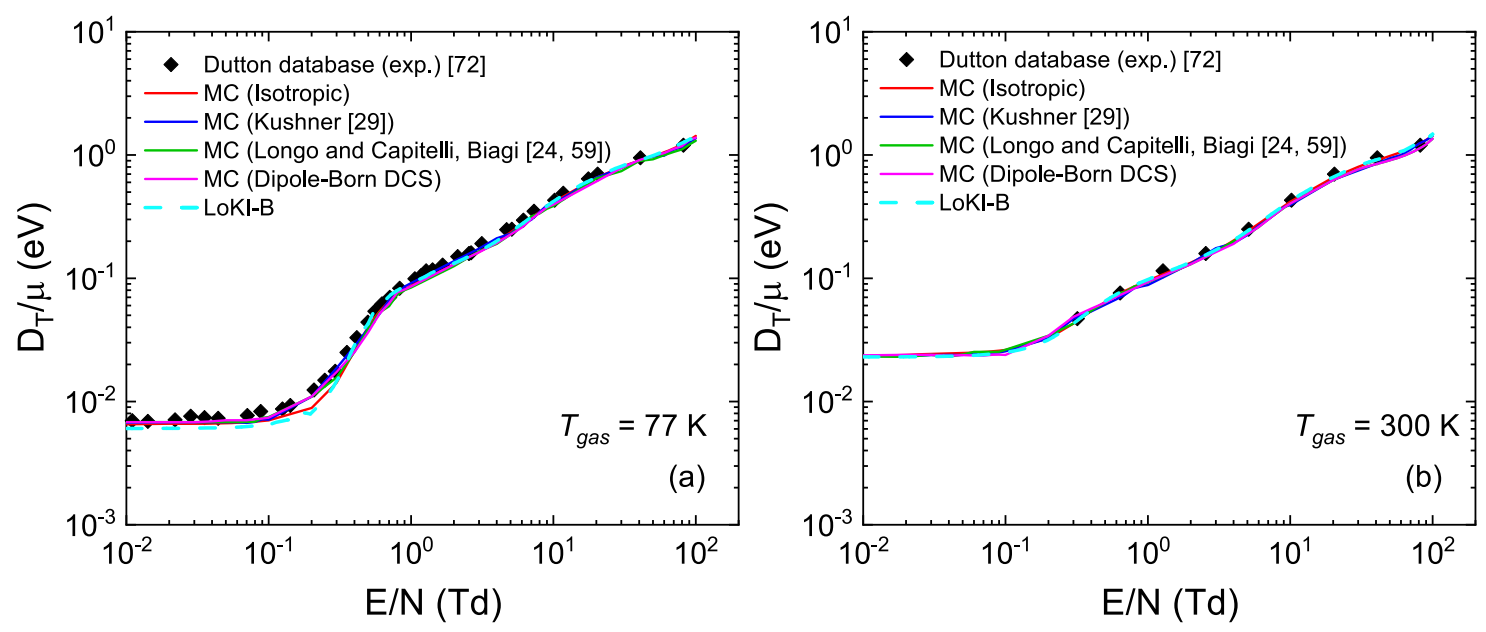

Figure 10. As in figure 8, but for the characteristic energy, with calculations using Biagi's cross sections set [24].

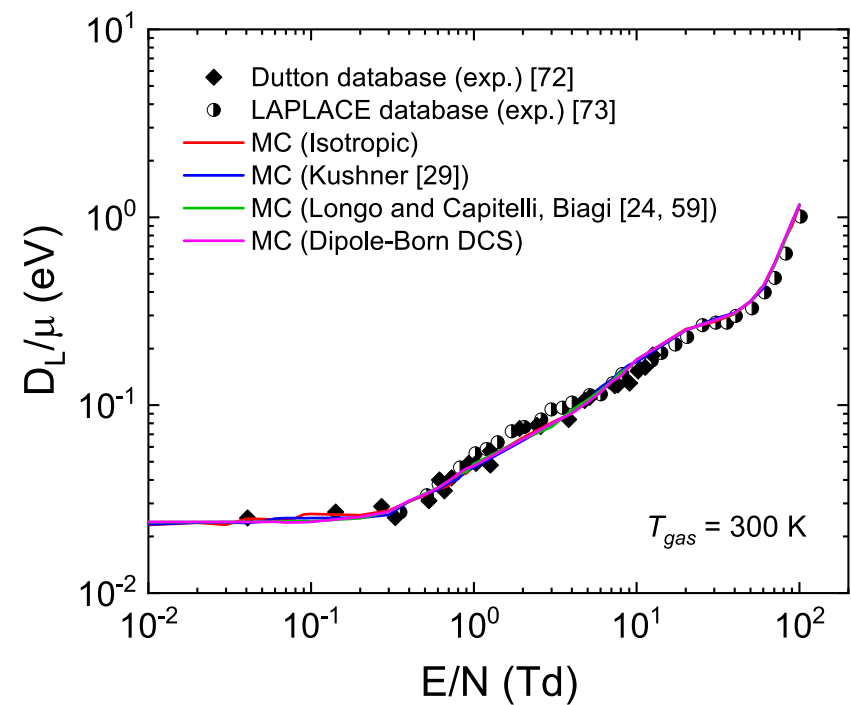

Figure 11. Longitudinal diffusion coefficients divided by electron mobilities at $300 \mathrm{~K}$ measured $[72,73]$ (points) and calculated with MC simulations (curves), using Biagi's cross sections set [24] and different angular scattering models for dipole rotational collisions, as in figure 8. MC isotropic (red curve), MC with Kushner's model [29] (blue curve), MC with Longo and Capitelli's model [59] (green curve) and MC with dipole-Born DCS model (magenta curve). A

Boltzmann distribution at $300 \mathrm{~K}$ for rotational and vibrational levels of the ground electronic state of $\mathrm{CO}$ is assumed.

approximation for the excitation from rotational quantum number $J$ to $J+2$ [37]:

$$
\sigma_{J, J+2}^{\text {quadr }}(\epsilon)=\frac{8 \pi}{15} Q^{2} a_{0}^{2}\left(\frac{\epsilon+V_{J, J+2}}{\epsilon}\right)^{1 / 2} \frac{(J+1)(J+2)}{(2 J+1)(2 J+3)},
$$

where $Q=1.86$ is the effective electric quadrupole moment of the CO molecule in units of $e a_{0}^{2}$ [74] and $V_{J, J+2}$ is the energy threshold for the transition $J \rightarrow J+2$. In this work, 13 different quadrupole excitations of rotational levels of the ground vibrational state have been considered, according to the selection rule $|\Delta J|=2$. Moreover, the corresponding de-excitation cross sections have been calculated from the Klein-Rosseland

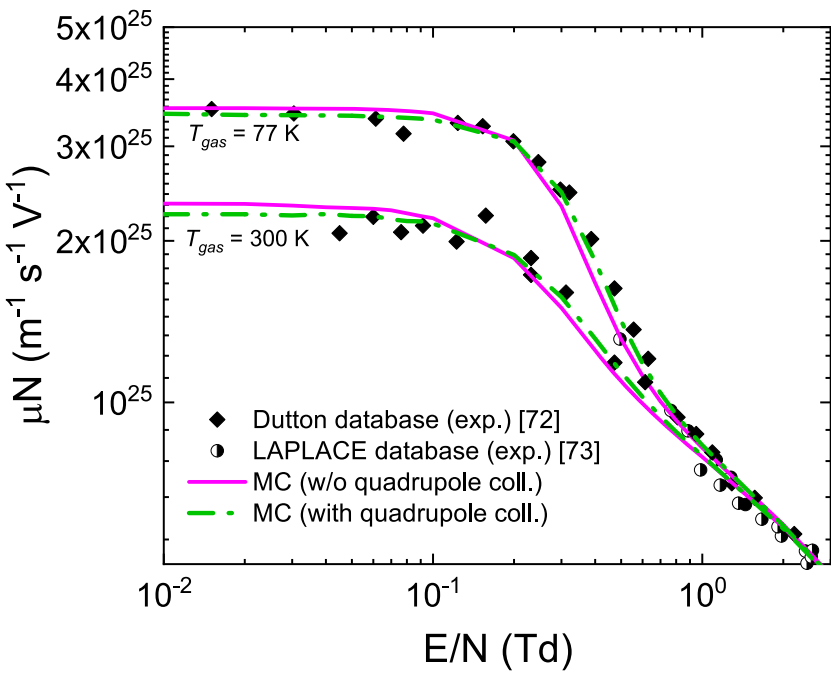

Figure 12. Reduced mobilities at $77 \mathrm{~K}$ and $300 \mathrm{~K}$ measured [72, 73] (points) and calculated (curves) from MC simulations, including (green curve) or neglecting (magenta) quadrupole rotational collisions in Biagi's cross sections set [24]. A Boltzmann distribution at $T_{\text {gas }}$ for rotational and vibrational levels of the ground electronic state of $\mathrm{CO}$ is assumed.

formula [35]. These cross sections are valid for low-energy electrons, since the main part of the interaction is long-range. For $\epsilon \geqslant 1.0 \mathrm{eV}$, where the cross sections become independent on the electron energy, an artificial Born decay of $1 / \epsilon$ was introduced. The angular distribution of the quadrupole rotational collisions is assumed to be isotropic. In fact, there is no forward peaking in the corresponding DCSs, as opposed to scattering by dipole potential [33].

In figure 12 , measurements of $\mu N$ at $77 \mathrm{~K}$ and $300 \mathrm{~K}$ $[72,73]$ are compared with the results from MC simulations, with and without the presence of quadrupole collisions in Biagi's cross sections set.

For $0.01 \leqslant E / N \leqslant 1 \mathrm{Td}$, differences between the calculated $\mu N$, including or neglecting quadrupole processes, are up to $8 \%$ and $5 \%$, at $77 \mathrm{~K}$ and $300 \mathrm{~K}$, respectively. These differences decrease with increasing $E / N$, becoming less than 
$0.5 \%$ for $E / N>1 \mathrm{Td}$. Results show that the inclusion of quadrupole processes improves the agreement between MC simulations and measurements of $\mu N$, for $E / N$ values between 0.2 and $0.8 \mathrm{Td}$. This is due to the fact that quadrupole contributions are beyond $10 \%$ for energies greater than $20 \mathrm{meV}$, being comparable to the dipole contributions at energies above 100 meV [9]. Hence, although neglected in most of the previous studies, quadrupole interactions are important to improve the agreement between measurements and calculations in the narrow region of $E / N$ values where transport parameters exhibit stronger variations. In section 6 , it is shown that this is the case also for the characteristic energy in addition to the electron reduced mobility.

\section{Implementation of the anisotropic treatment of rotational collisions in the two-term Boltzmann solver LoKI-B}

In this section, the treatment of dipole rotational collisions in LoKI-B [20] is extended by including anisotropic effects. A patch file including the changes with respect to the current LoKI-B implementation [67] is provided as supplementary information (https://stacks.iop.org/PSST/30/075001/mmedia). The extension follows the pioneering work by Reid [25], Kumar and co-authors [75] and Phelps and Pitchford [26], who introduced anisotropic terms in the two-term Boltzmann equation based on the Legendre polynomials expansion. In this work, the contribution of second-kind (superelastic) collisions has been considered as well. The treatment assumes that the angular dependence of the DCS is based only on the polar angle $\theta$ between the initial and the final relative velocity vectors in the laboratory frame.

According to Makabe and Petrovic [76], the DCS for the $k$ th collisional process can be expressed as an expansion in Legendre polynomials $P_{j}(\cos \theta)$ under azimuthal symmetry as

$$
\frac{\mathrm{d} \sigma_{k}(\epsilon, \theta)}{\mathrm{d} \Omega}=\sum_{j=0}^{\infty} \frac{2 j+1}{4 \pi} \sigma_{k}^{j}(\epsilon) P_{j}(\cos \theta),
$$

where the quantities $\sigma_{k}^{j}(\epsilon)$, hereafter termed partial cross sections, are the $j$ th Legendre polynomial coefficients of the expansion, for the $k$ th electron-CO molecule collision. Using the orthogonality relation of the polynomials, the partial cross sections can be expressed as [26]

$$
\sigma_{k}^{j}(\epsilon)=2 \pi \int_{0}^{\pi} \frac{\mathrm{d} \sigma_{k}(\epsilon, \theta)}{\mathrm{d} \Omega} P_{j}(\cos \theta) \sin \theta \mathrm{d} \theta .
$$

The relation of $\sigma_{J, J+1}^{\mathrm{ICS}}(\epsilon)$ and $\sigma_{J, J+1}^{\mathrm{MT}}(\epsilon)$, for the rotational transition $J \rightarrow J+1$, with the corresponding partial cross section $\sigma_{J, J+1}^{j}(\epsilon)$ (defined by equation (32)) is trivial, if the definitions of ICS and MT cross sections, given by equations (2) and (5), respectively, are taken into account. Hence, by truncation of the DCS expansion (equation (31)) to the first order, it can be noted that

$$
\sigma_{J, J+1}^{\mathrm{ICS}}(\epsilon) \simeq \sigma_{J, J+1}^{0}(\epsilon)
$$

and

$$
\sigma_{J, J+1}^{\mathrm{MT}}(\epsilon) \simeq \sigma_{J, J+1}^{0}(\epsilon)-\sigma_{J, J+1}^{1}(\epsilon),
$$

where equation (33) reveals that the zeroth-order partial cross section $\sigma_{J, J+1}^{0}(\epsilon)$ for the rotational collision $J \rightarrow J+1$ is equal to the corresponding ICS, and equation (34) relates the zeroth and the first order partial cross sections with the corresponding MT cross section. It should be noted that equation (34) is an approximation for the case of rotational collisions, valid for small relative differences in the electron speed before and after the collision [25]. From equations (33) and (34), it can be inferred that the only information on the angular dependence of the scattering, retained from a two-term expansion of the DCS in Legendre polynomials, is related to the ICSs and the MT cross sections.

The two-term expansion of the EVDF in Legendre polynomials allows one to write the electron Boltzmann equation as a system of two coupled differential equations, representing particle/energy conservation and momentum conservation [75], the latter corresponding to [20]

$$
-\epsilon \frac{E}{N} \frac{\mathrm{d} f_{0}(\epsilon)}{\mathrm{d} \epsilon}=C\left[f_{1}\right],
$$

where $C\left[f_{1}\right]$ is the collision operator for the anisotropic component $\left(f_{1}\right)$ of the EVDF of the form:

$$
C\left[f_{1}\right]=\sum_{i} J_{i}^{\text {ela. }}(\epsilon)+\sum_{i, j>i}\left(J_{i, j}^{\text {exc. }}(\epsilon)+J_{j, i}^{\text {de }- \text { exc. }}(\epsilon)\right) .
$$

Here, $J_{i}^{\text {ela. }}(\epsilon)$ is the anisotropic component of the operator describing elastic collisions of electrons with the $i$ th state of the gas, $J_{i, j}^{\text {exc. }}(\epsilon)$ is the anisotropic component of the operator describing electron collisions for the excitation of an arbitrary level $i$ to an arbitrary level $j$, and $J_{j, i}^{\mathrm{de}-\text { exc. }}(\epsilon)$ is the corresponding operator for the de-excitation of $j \rightarrow i$. Note that equation (36) can be trivially extended to account for the effects of non-conservative electron collisions and/or to the case of an arbitrary gas mixture [20]. In a general case, the previous operator can be expressed as

$$
\begin{aligned}
J_{i}^{\text {ela. }}(\epsilon)= & \delta_{i}\left[\epsilon \sigma_{i, \text { ela }}^{\mathrm{MT}}(\epsilon) f_{1}(\epsilon)\right], \\
J_{i, j}^{\text {exc. }}(\epsilon)= & \delta_{i}\left[\epsilon \sigma_{i, j}^{0}(\epsilon) f_{1}(\epsilon)-\left(\epsilon+V_{i, j}\right) \sigma_{i, j}^{1}\left(\epsilon+V_{i, j}\right)\right. \\
& \left.\times f_{1}\left(\epsilon+V_{i, j}\right)\right], \\
J_{j, i}^{\text {de }- \text { exc. }}(\epsilon)= & \delta_{j} \frac{g_{i}}{g_{j}}\left[\left(\epsilon+V_{i, j}\right) \sigma_{i, j}^{0}\left(\epsilon+V_{i, j}\right) f_{1}(\epsilon)\right. \\
& \left.-\epsilon \sigma_{i, j}^{1}(\epsilon) f_{1}\left(\epsilon-V_{i, j}\right)\right],
\end{aligned}
$$

where $\delta_{i}=N_{i} / N$ is the fractional number density of a generic state $i$ relative to the total gas density, $\sigma_{i, \text { ela }}^{\mathrm{MT}}$ is the elastic MT 
cross section for electron collisions with the $i$ th state of the gas, and $\sigma_{i, j}^{0}$ and $\sigma_{i, j}^{1}$ are the partial cross sections for the electron impact excitation between states $i$ and $j$ with energy threshold $V_{i, j}$ and statistical weights $g_{i}$ and $g_{j}$, respectively. Note that, in equation (39), micro-reversibility is assumed in order to express the de-excitation cross sections as a function of the corresponding excitations [35]. Often, inelastic cross sections are small when compared to elastic ones [25], in which case one can neglect the $J_{i, j}^{\text {exc. }}(\epsilon)$ and $J_{j, i}^{\text {de-exc. }}(\epsilon)$ terms in equation (36). However, this assumption is not always accurate in molecular gases, where the inelastic (rotational and vibrational) collisions may contribute significantly to the net exchange of momentum.

By substitution of equations (37)-(39) into equations (36) and (35) can be rewritten as proposed by Shkarofsky [77]

$$
f_{1}(\epsilon)=-\frac{E}{N} \frac{1}{\Omega_{\mathrm{c}}(\epsilon)} \frac{\mathrm{d} f_{0}(\epsilon)}{\mathrm{d} \epsilon}
$$

where $\Omega_{\mathrm{c}}(\epsilon)$ represents a total (effective) electron-neutral cross section for MT, defined as

$$
\begin{aligned}
\Omega_{\mathrm{c}}(\epsilon)= & \left\{\sum_{i} \delta_{i} \sigma_{i, \mathrm{ela}}^{\mathrm{MT}}(\epsilon)+\sum_{i, j>i}\left[\delta_{i} \sigma_{i, j}^{0}(\epsilon)\right.\right. \\
& \left.\left.+\delta_{j} \frac{g_{i}}{g_{j}} \frac{\epsilon+V_{i, j}}{\epsilon} \sigma_{i, j}^{0}\left(\epsilon+V_{i, j}\right)\right]\right\} \\
& -\left\{\sum_{i, j>i}\left[\delta_{i} \frac{\epsilon+V_{i, j}}{\epsilon} \sigma_{i, j}^{1}\left(\epsilon+V_{i, j}\right) \frac{f_{1}\left(\epsilon+V_{i, j}\right)}{f_{1}(\epsilon)}\right]\right\} \\
& -\left\{\sum_{i, j>i}\left[\delta_{j} \frac{g_{i}}{g_{j}} \sigma_{i, j}^{1}(\epsilon) \frac{f_{1}\left(\epsilon-V_{i, j}\right)}{f_{1}(\epsilon)}\right]\right\}
\end{aligned}
$$

As noted in [26], $\Omega_{\mathrm{c}}(\epsilon)$ is not uniquely defined, e.g. equation (41) is valid under a two-term expansion of the EVDF in Legendre polynomials. The terms on the right-hand-side of equation (41) represent, in order, the momentum exchange due to elastic collisions, electron impact excitations $i \rightarrow j$, and deexcitations $j \rightarrow i$. Note that the first term is equivalent to the one implemented in [20], under the assumption of isotropic excitation/de-excitation collisions leading to a pure loss of momentum after each event. More generally, if anisotropic scattering is a relevant part of the net momentum exchange, reentry terms due to excitations and de-excitations (i.e. the second and the third term on the right-hand side of equation (41), respectively) should also be taken into account. These terms are generally omitted in Boltzmann solvers based on the twoterm expansion in Legendre polynomials, due to the small ratio $f_{1}\left(\epsilon \pm V_{i, j}\right) / f_{1}(\epsilon)$.

In this study, the difference between ICSs and MT cross sections for dipole rotational collisions given by equations (3) and (6), evinces that the anisotropic scattering plays a relevant role on the calculations of electron swarm parameters. Hence, the full form of $\Omega_{\mathrm{c}}(\epsilon)$ in equation (41) must be adopted.

Unless the incident electron energy is very low, the difference between the electron energies before and after rotational collisions can be neglected, such that $\epsilon \pm V_{J, J \pm 1} \sim \epsilon$. Hence, $\Omega_{\mathrm{c}}(\epsilon)$ can be approximatively written, evidencing the contribution of the rotational collisions for MT, as

$$
\begin{aligned}
\Omega_{\mathrm{c}}(\epsilon) \simeq & \sum_{i} \delta_{i} \sigma_{i, \mathrm{ela}}^{\mathrm{MT}}(\epsilon)+\sum_{i, j>i}\left[\delta_{i} \sigma_{i, j}^{0}(\epsilon)\right. \\
& \left.+\delta_{j} \frac{g_{i}}{g_{j}} \frac{\epsilon+V_{i, j}}{\epsilon} \sigma_{i, j}^{0}\left(\epsilon+V_{i, j}\right)\right] \\
& +\sum_{J, J+1}\left[\delta_{J} \sigma_{J, J+1}^{\mathrm{MT}}(\epsilon)+\delta_{J+1} \frac{g_{J}}{g_{J+1}} \sigma_{J, J+1}^{\mathrm{MT}}(\epsilon)\right],
\end{aligned}
$$

where the first term represents the elastic contribution for MT, the second term is the inelastic contribution from all collisions (excitations and de-excitations) except for the dipole rotational collisions and the third term is the contribution of the dipole rotational excitations and de-excitations. From equation (42), it can be seen that the contribution for MT of all inelastic processes, except for dipole-rotational collisions, is described by ICSs, whereas electron-dipole interactions leading to rotational collisions are described by the corresponding MT cross sections. The advantage of using equation (42), with respect to equation (41), in a two-term Boltzmann solver is clear. In fact, due to the small energy between excited rotational levels, a local operator is employed for the calculation of inelastic and superelastic contributions to the electron energy fluxes (i.e. the third term on the right-hand-side of equation (42) depends only on $\epsilon$ and not on $\left.\epsilon \pm V_{J, J+1}\right)$. This treatment is also common for the description of rotational collisions under the continuous approximation, based on a generalization of the Fokker-Planck equation [78]. The applicability of equation (42), even for $E / N \leqslant 1 \mathrm{Td}$, where rotational collisions are dominant with respect to elastic ones, is justified for this work, due to the fact that the mean electron energy is always (at least) a factor 20 higher than the energy threshold for the excitation of the first rotational level. Thus, as shown in figure 2, the approximation introduced in the rotational MT cross section derived from equation (5) is negligible. Moreover, note that results obtained from the LoKI-B solver using equation (42) are in good agreement with the ones obtained from MC simulations, within a few percent, the latter not depending on an explicit definition of the rotational MT cross section. More generally, it is important to note that the assumptions underlying equation (42) are not applicable to any other inelastic collisions, including electron impact vibrational excitations, where the higher energy difference between excited vibrational levels leads to an invalidation of the local approximation. For these cases, using equation (41) is recommended, since it is based on the direct calculations of first-order partial cross sections from the DCS.

The previous analysis can be used to update the calculation results of the two-term solver LoKI-B. Our study shows that it is sufficient to extend the input data for the dipole rotational collisions providing, as before, the ICSs of equations (3) and (4) to calculate the collision operator of the isotropic equation, in addition to the MT cross sections of equations (6) and (7) to calculate the cross section of equation (42), used in the anisotropic equation (40). Moreover, the improved treatment of the anisotropic scattering for electrons in $\mathrm{CO}$ resolves the apparent inconsistencies at low energies between the total 

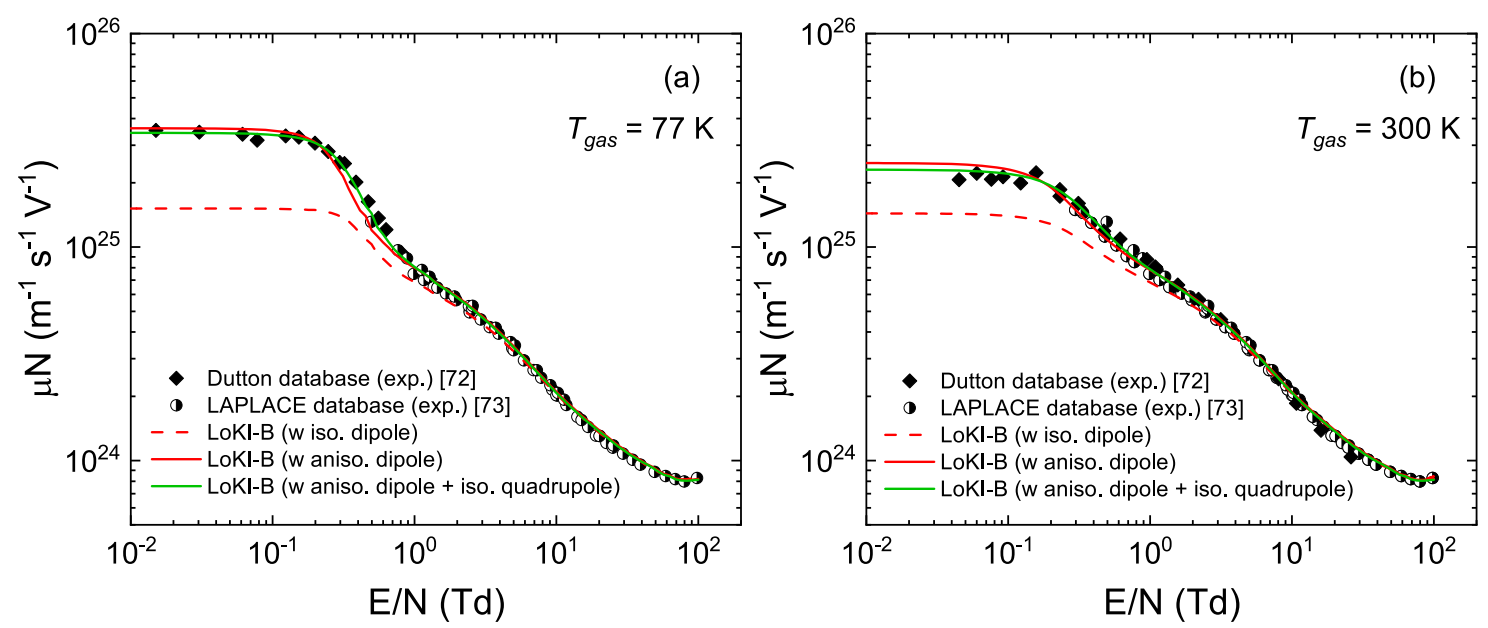

Figure 13. Reduced mobilities at $77 \mathrm{~K}$ (a) and $300 \mathrm{~K}$ (b) measured (points) [72, 73] and calculated (curves). Calculations were obtained using LoKI-B [20], with Biagi's cross sections [24] and different scattering assumptions for dipole and quadrupole rotational collisions. LoKI-B with isotropic scattering (red dashed curve), anisotropic scattering in dipole rotational collisions (solid red curve) and anisotropic scattering in dipole rotational collisions with the inclusion of isotropic scattering for quadrupole collisions (solid green curve). A Boltzmann distribution at $T_{\text {gas }}$ for rotational and vibrational levels of the ground electronic state of $\mathrm{CO}$ is assumed.
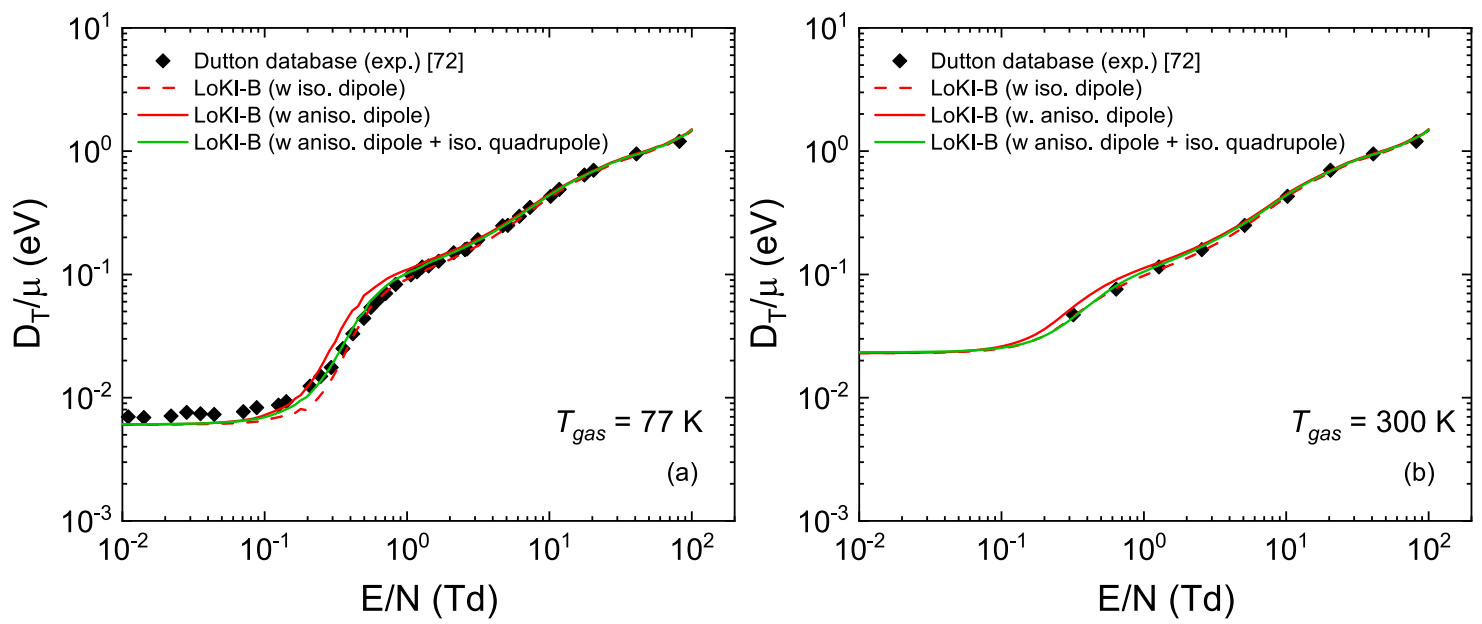

Figure 14. As in figure 13, but for the characteristic energy.

MT and the sum of all ICSs for rotational collisions, published in the Phelps database of LXCat [16], and described by Ogloblina and co-authors [18]. In fact, as already suggested in section 3, the scattering of low-energy electrons in $\mathrm{CO}$ embeds an important contribution from dipole rotational collisions, described by anisotropic DCSs, responsible for MT cross sections smaller than the corresponding ICSs.

The new calculation results in CO, obtained using LoKIB with the cross sections of Biagi [24] and the cross section of equation (42) (to account for the anisotropic scattering in dipole rotational collisions), are presented in figures 13 and 14 for the electron reduced mobility and the electron characteristic energy, respectively. Parts (a) and (b) of these figures show results at $77 \mathrm{~K}$ and $300 \mathrm{~K}$, respectively. The calculations assume the same working conditions as in section 5, as well as the same input data for the elastic MT and the vibrational/electronic excitation/de-excitation cross sections. For comparison purposes, the figures show also the values of the swarm parameters obtained experimentally [72, 73], and calculated (i) assuming the isotropic scattering approximation for dipole rotational collisions (as in the LoKI-B curves of figures 8 and 10), or (ii) further considering electricquadrupole rotational cross sections.

Figure 13 shows good agreement (within a few percent) between measurements and calculations, when anisotropic effects for dipole rotational collisions are included in LoKIB. In particular, the results for $\mu N$ from LoKI-B accounting for anisotropic dipole interactions agree within $2 \%$ with MC simulations assuming a dipole-Born angular scattering model (section 4.1). Moreover, the addition of electric-quadrupole interactions in LoKI-B contributes to an excellent agreement with experimental data (within $2 \%$ ), especially in the range $0.2 \leqslant E / N \leqslant 0.8 \mathrm{Td}$ at $77 \mathrm{~K}$ (figure 13(a)), where discrepancies up to $8 \%$ can still be found if only dipole rotational collisions are taken into account. As mentioned in section 5.2, isotropic scattering in quadrupole rotational collisions was 
assumed, due to the nature of the quadrupole interaction potential in the Born approximation [33]. Hence, as opposed to dipole interactions, cross sections for those collisions are included in the second term of equation (42).

Contrarily to the large differences found in the LoKI-B calculations of $\mu N$, when adopting different scattering assumptions, the numerical results of characteristic energies $D_{\mathrm{T}} / \mu$ are barely affected by these assumptions, as shown in figure 14, and already discussed in section 4 . Nevertheless, the addition of quadrupole rotational collisions is again fundamental, especially in the range $0.1 \leqslant E / N \leqslant 1.0 \mathrm{Td}$, to obtain excellent agreement with measurements, within $2 \%$.

The previous treatment allows one to include the effects of anisotropic scattering in a two-term Boltzmann solver as a modification of the cross section in equation (40) representing the loss of forward momentum for electrons drifting through a gas, under the influence of an electric field. To summarize, note that one of the differences between an MC simulation method and a Boltzmann solution of the EBE based on the two-term approximation is that the former approach does not require an expansion of the DCS in Legendre polynomials. Hence, any angular distribution can be accurately described by MC simulations without the need of an explicit definition of MT cross sections. For these reasons, both measurements and calculations of DCSs for electron collisions in molecular gases are very much needed for an improved description of electron kinetics, based on swarm analysis.

\section{Conclusions}

Electron scattering in $\mathrm{CO}$ has been investigated computationally via MC simulations and two-term solutions of the Boltzmann equation, adopting two different electron impact cross sections sets: the recently proposed IST-Lisbon set [18] and Biagi's set, extracted from the Magboltz code v11.10 [24]. Numerical results were compared with measurements of electron swarm parameters in CO [72, 73].

Despite the results of previous studies, suggesting that anisotropic scattering effects are generally small [26, 79], it was found that by adopting different angular scattering models for dipole-rotational collisions it is possible to induce strong modifications in the calculated electron swarm parameters, such as the reduced mobility. For this parameter, differences between measurements and MC simulations of up to $50 \%$ and $30 \%$ at $77 \mathrm{~K}$ and $300 \mathrm{~K}$, respectively, were obtained for different angular scattering models. Moreover, we also conclude that the calculation of electron swarm parameters in $\mathrm{CO}$ is more influenced by the choice of the angular scattering model than the adoption of the two-term approximation. Indeed by changing the angular distribution, while keeping the various ICSs unchanged, one affects also the corresponding MT cross sections, hence the electron transport parameters. An accurate treatment of the angular distribution in electron-molecule rotational collisions is expected to be important also in other diatomic or polyatomic molecules, such as nitric oxide (NO), ammonia $\left(\mathrm{NH}_{3}\right)$ and water vapour $\left(\mathrm{H}_{2} \mathrm{O}\right)$.

The limitations found in previous descriptions of the anisotropic scattering for rotational collisions, such as in
$[29,59]$, were overcome in this work by deriving a coherent angular scattering distribution from the dipole-Born DCS [31]. This novel angular scattering model can be used in MC simulations for describing the strongly forward-peaked nature of dipole rotational collisions between electrons and linear molecules.

The present investigation provided also important conclusions for improving the swarm derivation of electron scattering cross sections in general, and more specifically in the case of the $\mathrm{CO}$ molecule analysed here. On the one hand, the inclusion in MC simulations of the anisotropic scattering model based on the dipole-Born DCS, in combination with the Biagi's cross sections set [24], leads to good agreement (within a few percent) between measurements and simulations of electron transport parameters in CO. Notice that, despite a more recent version of Biagi's cross sections was released in the Magboltz code v11.11 [55], a general good agreement is already obtained by using cross sections from the previous version [24]. In fact, the set in Magboltz v11.10 [24] was already optimized for anisotropic scattering of dipole rotational collisions. The impact of the recent update of vibrational and electronic excitation cross sections on the calculations of electron swarm parameters requires future investigation.

On the other hand, using this novel scattering model with the IST-Lisbon dataset [18] leads to strong deviations between measurements and simulations. These deviations are expected, since the IST-Lisbon cross sections have been derived under the isotropic scattering assumption, not consistent with a Born treatment of the rotational DCS [21,33]. Nevertheless, this cross section set remains suited for solvers adopting isotropic scattering, and indeed it can be safely used in any solver for $E / N>1 \mathrm{Td}$. In any case, the present conclusions suggest the interest in proposing an alternative IST-Lisbon cross section set, so as to properly describe the anisotropic features of rotational collisions in $\mathrm{CO}$, and the corresponding electron-neutral total MT in solvers accepting this description.

In addition, the anisotropic inelastic/superelastic scattering in dipole rotational collisions was implemented in the twoterm solver LoKI-B [20] using coherent expressions for the corresponding ICSs and MT cross sections. The changes with respect to the current LoKI-B implementation [67] to include anisotropic effects in dipole rotational collisions are available as supplementary information with this work and they will be part of a future open-source version of the code. In particular, the information about the DCS is included in LoKI-B by an adequate modification of the MT cross section, representing the total momentum lost by the electrons in elastic and inelastic collisions with the background gas, as proposed by Reid [25] and Phelps and Pitchford [26]. This modification leads to a good agreement between LoKI-B calculations and measurements $[72,73]$ of the electron reduced mobility and characteristic energy, at low and moderate $E / N$ values, when adopting Biagi's cross sections.

For completeness, the inclusion of quadrupole rotational collisions in Biagi's cross sections set has been investigated. These processes were neglected in the previous studies by Hake and Phelps [13], and by Ogloblina and co-authors [18]. From the present study, it can be inferred that both the 
quadrupole and the dipole contributions are comparable at energies above $100 \mathrm{meV}$ and that the inclusion of the former improves the agreement between measurements and calculations of swarm parameters in the range $0.1 \leqslant E / N \leqslant 1 \mathrm{Td}$, correcting discrepancies of up to $8 \%$.

The present work highlights the importance of anisotropic scattering in low energy threshold rotational collisions and its effect on the calculation of electron swarm parameters in $\mathrm{CO}$. Our conclusions evince the importance of adopting a correct description for the angular scattering of electrons by molecules, hence reinforcing the need for accurate measurements and/or quantum-mechanical calculations of DCS.

\section{Acknowledgments}

The authors would like to express their gratitude to Professor S F Biagi from the University of Liverpool for useful discussions about electron impact cross sections in CO. This work is part of the Shell-NWO/FOM initiative 'Computational sciences for energy research' of Shell and Chemical Sciences, Earth and Life Sciences, Physical Sciences, FOM and STW. This work was partially funded by the Portuguese 'FCT-Fundação para a Ciência e a Tecnologia', under projects UIDB/50010/2020 and UIDP/50010/2020.

\section{Data availability statement}

All data that support the findings of this study are included within the article (and any supplementary files).

\section{ORCID iDs}

L Vialetto (1D https://orcid.org/0000-0003-3802-8001

S Longo (D) https://orcid.org/0000-0002-5038-7659

P Diomede (D) https://orcid.org/0000-0002-4523-3049

V Guerra (D) https://orcid.org/0000-0002-6878-6850

L L Alves (1) https://orcid.org/0000-0002-2677-574X

\section{References}

[1] Raju G G 2005 Gaseous Electronics: Theory and Practice (Boca Raton, FL: CRC Press)

[2] von Bülow H and Schellhorn M 1993 Appl. Phys. Lett. 63 287-9

[3] Pietanza L D, Colonna G and Capitelli M 2017 Plasma Sources Sci. Technol. 26125007

[4] Guerra V, Silva T, Ogloblina P, Grofulović M, Terraz L, da Silva M L, Pintassilgo CD, Alves L L and Guaitella O 2017 Plasma Sources Sci. Technol. 26 11LT01

[5] Hammer B, Morikawa Y and Nørskov J K 1996 Phys. Rev. Lett. $762141-4$

[6] McGuire S D, Tibère-Inglesse A C and Laux C O 2016 J. Phys. D: Appl. Phys. 49485502

[7] Erdman P W and Zipf E C 1983 Planet. Space Sci. 31 317-21

[8] Prinn R G and Barshay S S 1977 Science 198 1031-4

[9] Randell J, Gulley R J, Lunt S L, Ziesel J-P and Field D 1996 J. Phys. B: At. Mol. Opt. Phys. 29 2049-58

[10] Trajmar S, Register D F and Chutjian A 1983 Phys. Rep. 97 219-356
[11] Brunger M J and Buckman S J 2002 Phys. Rep. 357 215-458

[12] Petrović Z L, Dujko S, Marić D, Malović G, Nikitović ŽSǎšić O, Jovanović J, Stojanović V and Radmilović-Radjenović M 2009 J. Phys. D: Appl. Phys. 42194002

[13] Hake R D Jr and Phelps A V 1967 Phys. Rev. 15870

[14] Land J E 1978 J. Appl. Phys. 49 5716-21

[15] Itikawa Y 2015 J. Phys. Chem. Ref. Data 44013105

[16] 2020 Phelps database www.lxcat.net (last access 13 October 2020)

[17] 2020 Itikawa database www.lxcat.net (last access 13 October 2020)

[18] Ogloblina P, Tejero-del-Caz A, Guerra V and Alves L L 2020 Plasma Sources Sci. Technol. 29015002

[19] 2020 IST-Lisbon database www.lxcat.net (retrieved on 31 July 2020)

[20] Tejero-del-Caz A, Guerra V, Gonçalves D, da Silva M L, Marques L, Pinhão N, Pintassilgo C D and Alves L L 2019 Plasma Sources Sci. Technol. 28043001

[21] Altshuler S 1957 Phys. Rev. 107 114-7

[22] Vialetto L, Longo S and Diomede P 2019 Plasma Sources Sci. Technol. 28115015

[23] Vialetto L, Viegas P, Longo S and Diomede P 2020 Plasma Sources Sci. Technol. 29115006

[24] unpublished cross sections extracted from http://magboltz.web. cern.ch/magboltz/ (last access 10 September 2020)

[25] Reid I D 1979 Aust. J. Phys. 32 231-54

[26] Phelps A V and Pitchford L C 1985 Phys. Rev. A 31 2932-49

[27] Janssen J F J, Pitchford L C, Hagelaar G J M and van Dijk J 2016 Plasma Sources Sci. Technol. 25055026

[28] Casey M J E, Cocks D G, Boyle G J, Brunger M J, Dujko S, de Urquijo J and White R D 2019 Plasma Sources Sci. Technol. 28115005

[29] Kushner M J 1987 J. Appl. Phys. 61 2784-94

[30] Pitchford L C, ONeil S V and Rumble J R Jr 1981 Phys. Rev. A 23 294-304

[31] Crawford O H 1967 J. Chem. Phys. 47 1100-4

[32] Takayanagi K 1966 J. Phys. Soc. Japan 21 507-14

[33] Shimamura I and Takayanagi K 2013 Electron-Molecule Collisions (Berlin: Springer)

[34] Makabe T and White R 2015 J. Phys. D: Appl. Phys. 48485205

[35] Fowler R H 1924 London, Edinburgh Dublin Phil. Mag. J. Sci. 47 257-77

[36] Takayanagi K and Itikawa Y 1970 Adv. At. Mol. Phys. 6 105-53

[37] Gerjuoy E and Stein S 1955 Phys. Rev. 97 1671-9

[38] Crawford O H and Dalgarno A 1971 J. Phys. B: At. Mol. Phys. 4 494-502

[39] Jain A and Norcross D W 1992 Phys. Rev. A 45 1644-56

[40] Itikawa Y and Mason N 2005 Phys. Rep. 414 1-41

[41] Faure A, Gorfinkiel J D and Tennyson J 2004 Mon. Not. R. Astron. Soc. 347 323-33

[42] Garrett W R 1972 Mol. Phys. 24 465-87

[43] Itikawa Y and Takayanagi K 1969 J. Phys. Soc. Japan 26 1254-64

[44] Haddad G and Milloy H 1983 Aust. J. Phys. 36 473-84

[45] Petrovic Z and Crompton R 1989 Aust. J. Phys. 42 609-26

[46] Nakamura Y 1987 J. Phys. D: Appl. Phys. 20 933-8

[47] Saelee H T and Lucas J 1977 J. Phys. D: Appl. Phys. 10 343-54

[48] Pack J L, Voshall R E and Phelps A V 1962 Phys. Rev. 127 2084-9

[49] Kanik I, Trajmar S and Nickel J C 1993 J. Geophys. Res. 98 7447-60

[50] Sawada T, Sellin D L and Green A E S 1972 J. Geophys. Res. 77 4819-28

[51] Gibson J C, Morgan L A, Gulley R J, Brunger M J, Bundschu C T and Buckman S J 1996 J. Phys. B: At. Mol. Opt. Phys. 29 3197-214

[52] Ehrhardt H, Langhans L, Linder F and Taylor H S 1968 Phys. Rev. 173222 
[53] Laporta V, Cassidy C M, Tennyson J and Celiberto R 2012 Plasma Sources Sci. Technol. 21045005

[54] Swanson N, Celotta R J, Kuyatt C E and Cooper J W 1975 J. Chem. Phys. 62 4880-8

[55] http://magboltz.web.cern.ch/magboltz/ (last access 25 February 2021)

[56] Okhrimovskyy A, Bogaerts A and Gijbels R 2002 Phys. Rev. E $\mathbf{6 5} 037402$

[57] Allan M 2010 Phys. Rev. A 81042706

[58] Surendra M, Graves D B and Jellum G M 1990 Phys. Rev. A 41 $1112-25$

[59] Longo S and Capitelli M 1994 Plasma Chem. Plasma Process. $141-13$

[60] Capitelli M, Celiberto R, Colonna G, Esposito F, Gorse C, Hassouni K, Laricchiuta A and Longo S 2015 Fundamental Aspects of Plasma Chemical Physics: Kinetics vol 85 (Berlin: Springer)

[61] Skullerud H R 1968 J. Phys. D: Appl. Phys. 1 1567-8

[62] Longo S 2006 Plasma Sources Sci. Technol. 15 S181-8

[63] Longo S and Diomede P 2004 Eur. Phys. J. Appl. Phys. 26 $177-85$

[64]Simko T 1997 Modélisation du transport ionique et électronique dans un gaz soumis à un champ électrique. Comparaison modèle-expérience pour l'hydrogène $P h D$ Thesis Université de Paris-Sud

[65] Boeuf J-P 1985 Modélisation de la cinétique électronique dans un gaz faiblement ionisé PhD Thesis Université de Paris-Sud
[66] Vass M, Korolov I, Loffhagen D, Pinhão N and Donkó Z 2017 Plasma Sources Sci. Technol. 26065007

[67] https://github.com/IST-Lisbon/LOKI (last access 20 October 2020)

[68] Blevin H and Fletcher J 1984 Aust. J. Phys. 37 593-600

[69] White R D, Robson R E, Dujko S, Nicoletopoulos P and Li B 2009 J. Phys. D: Appl. Phys. 42194001

[70] Capitelli M, Ferreira C M, Gordiets B F and Osipov A I 2013 Plasma Kinetics in Atmospheric Gases vol 31 (Berlin: Springer)

[71] Pitchford L C and Phelps A V 1982 Phys. Rev. A 25 540-54

[72] 2020 Dutton database www.lxcat.net (retrieved on 31 July 2020)

[73] 2020 LAPLACE database www.lxcat.net (retrieved on 31 July 2020)

[74] Stogryn D E and Stogryn A P 1966 Mol. Phys. 11 371-93

[75] Kumar K, Skullerud H and Robson R 1980 Aust. J. Phys. 33 343-448

[76] Makabe T and Petrović Z L 2014 Plasma Electronics: Applications in Microelectronic Device Fabrication (Boca Raton, FL: CRC Press)

[77] Shkarofsky I P, Johnston T W and Bachynski M P 1966 The Particle Kinetics of Plasmas (Reading, MA: Addison-Wesley Developers Press)

[78] Ridenti M A, Alves L L, Guerra V and Amorim J 2015 Plasma Sources Sci. Technol. 24035002

[79] Haddad G N and Elford M T 1979 J. Phys. B: At. Mol. Phys. 12 L743-6 